\title{
Francesco Colonnas Traumwelt: Die Hypnerotomachia Poliphili
}

\section{1 Historische Notizen}

Die Erstausgabe der Hypnerotomachia Poliphili ${ }^{1}$ erschien 1499 bei Aldus Manutius in Venedig als illustrierte Prachtausgabe und galt lange Zeit als das schönste Buch aller Zeiten. ${ }^{2}$ Weder der Autor noch der Illustrator können eindeutig identifiziert werden, weshalb die Frage nach dem Autor einen grossen Teil der literaturwissenschaftlichen Forschung einnimmt. Gemutmasst wird über « Zuschreibungen von Alberti ${ }^{3}$ bis Aldus Manutius, von Lelio Cosmico bis Lorenzo de' Medici (um nur die Bekanntesten zu nennen) ». ${ }^{4}$ Wie für Thomas

1 Ich verwende folgende Ausgaben: Für den Originaltext (in der Folge abgekürzt mit $H P$ ): Francesco Colonna, Hypnerotomachia Poliphili, a cura di Marco Ariani e Mino Gabriele, Tomo primo: riproduzione dell'edizione aldiana del 1499, Milano 2010. Eine neuitalienische Nacherzählung findet sich in: Francesco Colonna, Hypnerotomachia Poliphili, a cura di Marco Ariani e Mino Gabriele, Tomo secondo: introduzione, traduzione e commento, Milano 2010. Für die deutsche Übersetzung: Francesco Colonna, Hypnerotomachia Poliphili, übersetzt und kommentiert von Thomas Reiser, 1. Auflage der Interlinearkommentarfassung, Breitenbrunn 2014. Reiser arbeitet mit den Originalseitenzahlen, die auch Ariani und Gabriele verwenden, entsprechend verwende ich zur Stellenangabe sowohl für den italienischen wie auch den deutschen Text die Abkürzung HP. Weiter verzichte ich darauf, die kunstvolle Typographie wiederzugeben, und zitiere mit Blocksatz. Selbstverständlich spielt die Typographie eine wichtige Rolle, insbesondere für die visuelle Wirkung des Textes, eine Analyse von dieser war im Umfang dieser Arbeit aber nicht möglich.

2 Liane Lefaivre, Leon Battista Alberti's "Hypnerotomachia Poliphili“: Re-Cognizing the Architectural Body in the Early Italian Renaissance, Cambridge Massachusetts 1997, S. 18.

3 Neben historischen Inkongruenzen und dem Mangel an Belegdokumenten dient auch die Betrachtung des Staunens dazu, die These, Alberti sei der Autor der $H P$, zu stürzen. Kretzulesco-Quaranta oder Lefaivre postulieren eine quasi absolute Nähe der $H P$ zu Albertis Zehn Bücher über die Baukunst, übersehen aber, dass das wichtigste ästhetische Prinzip der $H P$, das Staunen, in Albertis Traktat quasi inexistent - und wenn seltenerweise existent, dann zu bestimmten Zwecken funktionalisiert - ist. Albertis Architekturtheorie ist funktional getrieben, während das Funktionale in der $H P$ keine Rolle spielt, ja geradezu ausgeklammert wird.

4 Thomas Reiser, Einleitendes zur ,Hypnerotomachia Poliphili, in: Colonna, Hypnerotomachia Poliphili, S. v. Für eine Zuschreibung an Alberti siehe LEFaIVre, Leon Battista Alberti; ebenso Emanuela Kretzulesco-Quaranta, Les jardis du songe. „Poliphile" et la Mystique de la Renaissance, préface de Pierre Lyautey, Paris ${ }^{2} 1986$, S. 27. Für Lelio Cosmico argumentiert Roswitha Stewering, Architektur und Natur in der 
Reiser und Ariani/Gabriele, die sich auf Ciapponi / Pozzi ${ }^{5}$ und Casella ${ }^{6}$ stützen, scheinen mir die Argumente zugunsten des Kanonikers Francesco Colonna (1433/34-1527) am stichhaltigsten: ${ }^{7}$ Aus den Kapitelanfängen der $H P$ wurde das Akrostichon Poliam Frater Franciscus Columna ${ }^{8}$ Peramavit (,Polia liebte der Bruder Francesco Sehr') eruiert. Der Dominikanerorden, dem Francesco Colonna angehörte, war kulturell offen und « gestattete seinen Mitgliedern in den humanistischen Studien zahlreiche Freiheiten $»{ }^{9}$

In einem Wiegendruck der $H P$, auf den man 1723 im Konvent der Dominikaner zu Venedig stieß, findet sich indes ein Eintrag vom 20. Juni 1521, der besagt, dass jener Francesco Colonna des Akrostichons ein Mitglied des Predigerordens aus der Lagunenstadt gewesen sei, welcher sich aus Liebe zu einer gewissen ,Hippolita' (wohl volkstümlich für Polia) nach Treviso begeben hätte. ${ }^{10}$

Die Verbindung zu Treviso findet sich auch im Text, wenn Polia im zweiten Buch ihre Familie als (mythische) Gründerfamilie Trevisos nennt. Treviso wird am Ende des zweiten Buches als Ort der Niederschrift genannt («Taurisii », $H P, 465)$, zudem weisen einzelne sprachliche Elemente auf den Veneto hin. ${ }^{11}$

„Hypnerotomachia Poliphili“ (Manutius 1499) und Die Zuschreibung des Werkes an Niccolò Lelio Cosmico, Hamburg 1996.

5 Francesco Colonna, Hypnerotomachia Poliphili, edizione critica e commento a cura di Giovanni Pozzi e Lucia A. Ciapponi, 2 Bde., Padova 1968.

6 Maria Teresa Casella und Giovanni Pozzi, Francesco Colonna. Biografia e opere, 2 Bde., Padova 1959.

7 REISER, Einleitendes, S. vi. Wie schon erwähnt, ist auch die Identität des Illustrators nicht bekannt. Gemutmasst wird unter anderem über Bernardino Pinturicchio, Giovanni Bellini, Benedetto Montagna, Benedetto Mordone und Leon Battista Alberti. Siehe dazu die Einleitung von Marco Ariani und Mino Gabriele im zweiten Band ihrer Ausgabe (S. XCV-CIX).

8 «Columna » kann nicht nur als Nachname, sondern auch als Ablativ gelesen werden, das Akrostichon würde übersetzt dann folgendermassen lauten: „Bruder Francesco hat Polia voller Liebe mit dieser Columna geliebt“, wobei columna sowohl für Säule metaphorisch unter anderem für den vertikal angeordneten Text - als auch für das männliche Glied stehen kann. Siehe dazu Silvia Fogliati, Hypnerotomachia Poliphili, in: Dies. und Davide Dutto (Hgg.), Il giardino di Polifilo. Ricostruzione virtuale dalla Hypnerotomachia Poliphili di Francesco Colonna stampata a Venezia nel 1499 da Aldo Manuzio, Milano 2002, S. 11.

9 REISER, Einleitendes, S. vi.

10 Ebd., S. vi.

11 Eine ausführliche Studie zur aussergewöhnlichen Sprache der HP findet sich in PozzI, Francesco Colonna: Opere, S. $78-126$. 
Der Roman ${ }^{12}$ erzählt die Geschichte des Poliphilo, der in einem Traum im Traum mythische Orte durchwandelt und dort seiner Geliebten Polia - deren Zuneigung ihm im „wahren“ Leben verwehrt bleibt - begegnet. ${ }^{13}$ Ein Grossteil der Handlung spielt in der (Traum-)Natur, die entweder von antiken Baukunstwerken und Palästen durchsetzt oder als klassische Gartenkomposition angeordnet ist. ${ }^{14}$ Dabei ist es die spezifische Eigenheit der $H P$, die Liebeserzählung mit überaus ausladenden Kunstbeschreibungen aufzubrechen und zu transformieren. ${ }^{15}$ Die Kunstbeschreibungen sind quantitativ dominant und nicht für die Liebeserzählung funktionalisiert - beispielsweise als allegorischer Kommentar oder als ornamentaler Zusatz -, sondern als eigenständige Elemente der Erzählung konzipiert. ${ }^{16}$

12 In der Erzählung finden sich «Elemente des Märchens, der Utopie, der Idylle, des Dramas, der Dichtung und der Kunst- und Architekturtheorie und [sie] ist von heterogensten Quellen inspiriert [...] Aufgrund dieser Heterogenität ist am ehesten die Gattungsbezeichnung ,Roman‘ angebracht.» Siehe Dorothea Scholl, Groteske, Eros und Traum: Hypnerotomachia Poliphili, in: Dies., Von den „Grottesken“ zum Grotesken. Die Konstituierung einer Poetik des Grotesken in der italienischen Renaissance, Münster 2004, S. 237. Ich verwende den Begriff „Roman“ daher relativ generisch für die fiktive Langerzählung in Prosa.

13 Eine ausführliche Inhaltszusammenfassung findet sich in Fogliati, Hypnerotomachia Poliphili, S. 13-15.

$14 \mathrm{Zu}$ den Quellen der HP zählen theoretische Texte der Historiographie, Naturkunde, Rhetorik, Kunst- und Architekturtheorie wie auch literarische und satirische Werke. Von den antiken Autoren werden Plinius der Ältere, Apuleius, Ovid, Festus und Vergil am häufigsten zitiert, von den zeitgenössischen Texten sind es insbesondere die tre corone Dante, Petrarca und Boccaccio - sowie der Roman de la Rose von Guillaume de Lorris und Jean de Meun, die die $H P$ beeinflusst haben. Neben diesen Quellen dürfen die Texte Vitruvs und Albertis nicht vergessen werden; sie haben insbesondere das technischarchitektonische Vokabular Colonnas beeinflusst. Eine ausführliche Quellenanalyse findet sich in Lefaivre, Leon Battista Alberti, S. 56-62. Lefaivre ihrerseits verweist auf den Kommentarband von Pozzi / Ciapponi, S. 53-55, 323-338.

15 SteWE RING, Architektur und Natur, S. 14.

16 Ich weiche damit ab von der Interpretation Kirsten Dickhauts, die die Architekturdarstellungen als Sinnbild einer positiven Anthropologie und geselligen Utopie liest. Ich bin einverstanden, dass die Architekturwerke auf die Schöpfungskraft des Menschen verweisen und diesen dadurch positiv konnotieren. Der Bezug zu einer komplexen theoretischen und ethischen Gesellschaftsutopie und einer «christlich-naturrechtlich verankerte[n] Anthropologie» scheint mir allerdings von aussen an den Text herangetragen, denn dieser hebt weder das gesellige Leben noch ethisch-soziale Aspekte der Ehe zwischen Polia und Poliphilo - die Dickhaut als Bestätigung der Utopie des geselligen Miteinanders liest - speziell hervor. Im Gegenteil scheinen mir die Szenen, in denen geselliges (höfisches) Leben dargestellt wird, als Schauspiel inszeniert und damit wiederum als Kunstformen ausgestellt. Siehe Kirsten Dickhaut, Positives Menschenbild und venezianità. Kythera als Modell einer geselligen Utopie in Literatur und Kunst von der italienischen Renaissance bis zur französischen Aufklärung, Wiesbaden 2012, S. $168 \mathrm{ff}$. 
Die Hypnerotomachia, wenigstens in ihrem ersten Teil, ist demnach nicht eigentlich eine kontinuierliche Erzählung, sondern eine diskontinuierliche Serie von Beschreibungen, Ekphrasen; und diese Ekphrasen stehen zueinander in einem Verhältnis der Variation. [...] Die Ekphrasis, die im Epos, im antiken und mittelalterlichen Roman nur an privilegierten Stellen die Erzählung aufhält, wird hier zu deren Bau-Element, oder vielmehr: sie tritt an ihre Stelle. ${ }^{17}$

Diese Aussage stimmt in ihrem Grundsatz, ist aber dahingehend zu präzisieren, dass Kontinuität der Erzählung von den Beschreibungen nie vollständig unterbrochen wird. Allerdings sind die deskriptiven Passagen teilweise derart lang, dass sie die Rahmenhandlung in den Hintergrund drängen.

Während die Liebeshandlung eher stereotyp und oberflächlich erzählt wird, gestalten sich die Architektur- und Kunstbeschreibungen als fantasiereiche, komplexe Ausfaltungen der verschiedenen Werkkategorien: der materiellen, der handwerklichen, der technischen, der geometrischen, der historischen, der mythologischen, der imaginativen und insbesondere der ästhetischen Ebene, wobei versucht wird, ebenjene Elemente zu fassen und zu beschreiben, die zum herausragenden ästhetischen Wert der Objekte beitragen. Die $H P$ konfrontiert uns weder mit einer neoplatonischen Ideenästhetik noch mit einer (materiellen) Naturästhetik, sondern mit einer Ästhetik der künstlerischen Imagination und Kunstfertigkeit.

Genauso fantasiereich und originell präsentiert sich die Sprache der HP. Sie mischt lateinisches und italienisches Vokabular zu ungewohnten Wortschöpfungen und verwendet eine eigene, sich deutlich von der Alltagssprache unterscheidende Syntax. ${ }^{18}$ Damit demonstriert auch die Sprache

17 Gerhard Goebel, Erträumte Architektur: Francesco Colonnas Hypnerotomachia Poliphili, in: De rs., Poeta faber. Erdichtete Architektur in der italienischen, spanischen und französischen Literatur der Renaissance und des Barock, Heidelberg 1971, S. 67. Goebel erkennt eine Ähnlichkeit zu Polizianos Stanze della giostra, hebt jedoch den « Reichtum an konkreten Details und die Konstruktivität der Beschreibung », zudem das « Variationsverhältnis, in dem die beschreibenden Sequenzen zueinander stehen», als unterscheidende Faktoren hervor.

18 Andrea Elmer, Die Hypnerotomachia Poliphili als (vergessenes) Paradigma einer Ästhetik des Staunens, in: Johannes Bartuschat et al. (Hgg.), Poetiken des Staunens. Narratologische und dichtungstheoretische Perspektiven, München 2019, S. 193: « Augenfälligstes Merkmal der Hypnerotomachia ist die eigentümliche Sprache. Sie zeigt ein Ineinander von volkssprachiger Grammatik und lateinischem Wortschatz - zusätzlich angereichert mit griechischen Begriffen und Hieroglyphen wie auch mit zahlreichen Neologismen. Ein Grossteil der Sätze gestaltet sich elliptisch in- und aneinandergeschachtelt und weist ,orientalisierende[...] und antikisierende[...] Konstruktionen' auf. Dorothea Scholl spricht treffend von einem ,irrealen Idiom[...] ausserhalb von Raum und Zeit', das den Traum-Zeit-Raum der Erzählung konstituiert. Pozzi fasst die ,poetischen und sprachlichen Strukturen' mit dem Prinzip der ,Kontamination'. Der Roman wird in der 1. Person 
den ausserordentlichen Imaginations- und Kreationsreichtum des Künstleringeniums. An Letzteres ist das Staunen als zentrales Motiv gebunden. Es charakterisiert vornehmlich die Kunstbetrachtung, während der Liebeshandlung andere Attribute, beispielsweise die dolcezza, zukommen.

\section{2 $\quad$ Kontext der Forschung}

Als erster Buchdruck mit Holzschnitten wurde die $H P$ insbesondere druckund kunsthistorisch rezipiert. Zudem hat sie die Architektur der Renaissance, allen voran die Gartenarchitektur, nachhaltig beeinflusst. ${ }^{19}$ Demgegenüber sind die literaturwissenschaftlichen Forschungen überschaubar: Neben den genannten kritischen Ausgaben, zwei Bänden über Leben und Werk, der deutschen Interlinearversion sowie einzelnen Spezialstudien ${ }^{20}$ gibt es relativ wenige relevante Forschungsergebnisse. ${ }^{21}$

Die $H P$ wird häufig als allegorisches, enigmatisches oder alchemistisches Werk gelesen - wohl motiviert durch das Akrostichon, die eigentümliche Sprache sowie die mythisch-fantastische Traumwelt. So interpretiert beispielsweise Emanuela Kretzulesco-Quaranta - in ihrer Untersuchung der Gartendarstellungen sowie deren Einfluss auf die Gartenkunst der Renaissance - Poliphilo als «Visionnaire-Narrateur», der durch die antik anmutende Landschaft wandelt, in der Hoffnung, an den Ursprung der Welt und des Lebens zurückzugelangen. ${ }^{22}$ Kretzulesco-Quaranta unterschlägt damit zweierlei: Das Einzige, was Poliphilo zu ergründen sucht, ist die Beschaffenheit der Kunstwerke.

erzählt, was eine Identifikation des Lesers mit dem Protagonisten begünstigt - und was gerade auch für das Staunen und dessen Übertragung auf den Leser von Relevanz ist. » Ich beziehe mich in diesem Absatz auf ScholL, Groteske, Eros und Traum, S. 231, und verweise auf die ausführlichen Sprachstudien von Giovanni Pozzi und Giongio Agamben: Pozzi, Francesco Colonna: Opere, S. 78-126; ebenso Giongio Agamben, Il sogno della lingua. Per una lettura del Polifilo, in:Vittore Branca, Carlo Ossola und SALOMon Resnik (Hgg.), I linguaggi del sogno, Firenze 1984, S. 417-430.

$19 \mathrm{Zu}$ den berühmtesten an die $H P$ angelehnten Kunstwerken zählen der Obelisco della Minerva (Piazza della Minerva, Rom) von Giovan Lorenzo Bernini (fertiggestellt von Ercole Ferrata) sowie der Sacro Bosco in Bomarzo, der von Vicinio Orsini in Auftrag gegeben wurde. Auch die Gärten der Villa von Cosimo de' Medici in Castello, der Villa Francesco de' Medici in Pratolino, der Villa Aldobrandini in Frascati sowie die BoboliGärten zeigen sich von der Traumlandschaft der Hypnerotomachia inspiriert. Siehe dazu SCHOLL, Groteske, Eros und Traum, S. 238.

20 Besonders nennenswert sind die Studien von Dorothea Scholl und Gerhard Goebel: SCHoll, Groteske, Eros und Traum, S. 223-287; Goe B EL, Erträumte Architektur, S. 38-68.

21 Zum selben Schluss kommt Dickhaut, Positives Menschenbild, S. 149.

22 Kretzulesco-Quaranta, Les jardis du songe, S. 2 O. 
Die $H P$ beschreibt weder eine Initiation, noch gelangt Poliphilo am Schluss zu einem metaphysischen Ziel. Im Gegenteil, er wacht desillusioniert aus dem Traum auf, um einige Kunstbetrachtungen reicher, aber ohne eine höhere Kenntnis der « sorgente della Vita » erlangt zu haben, und insbesondere ohne seine Geliebte. ${ }^{23}$

Genauso wie die Traumlandschaft wurde auch die Liebesgeschichte teleologisch gedeutet. So deutet Mino Gabriele den Roman als Kampf Poliphilos gegen die «körperlichen Schlingen » hin zur reinen Liebe, als Bewegung von der «niedrigen Welt» zu den « unsterblichen Dingen ». ${ }^{24}$ Diesen Kampf vollziehe Poliphilo über drei Stufen: zuerst über eine infantile und irrationale Leidenschaft, gefolgt von der «Freigiebikeit der Liebe» und schliesslich einer irdischen und einer himmlischen Manifestation der voluptas. ${ }^{25} \mathrm{Im}$ Text lässt sich ein derartiger ascensus animce allerdings kaum belegen; wie bereits erwähnt, bleibt Polia einzig als (Körper-)Bild interessant, ${ }^{26}$ eine andere, „höhere" Qualität der Liebe zu ihr wird nicht erwähnt. Die Liebeshandlung endet in der körperlichen Vereinigung der Liebenden und in der Betrachtung von Polias «vergöttlichte[m] Abbild » - bevor Polia in einem « Rauchfaden » aus Moschus und Ambra verschwindet $(H P, 464)$.

Was mir hingegen von besonderer Bedeutung scheint, ist die häufige Nennung der voluptas in Zusammenhang mit der Betrachtung erstaunlicher Kunstwerke. Ist die voluptas im ersten Teil der HP noch vorwiegend als Vergnügen und Freude zu verstehen, konkurriert sie im zweiten Teil mit der erotischen Begierde, die auf Polia gerichtet ist:

Mirai daposcia cum singulare uoluptate una laquearia contignatione summamente fabre decora, in una æquabile planitie subtensa di pare \& comparatione priua, rendeua uno superbo cœlo, cum disposite interuallature di multiplice deformatione, cum tirata \& adlibellata dimensione $[\ldots]^{27}(H P, 118 ; 1$. Teil $)$

Tra gli quali [i seni di Polia] uolupticamente miraua una deliciosa uallecula, oue era la delicata sepultura dilalma mia. ${ }^{28}(H P, 240 ; 2$. Teil $)$

23 Fogliati, Hypnerotomachia Poliphili, S. 11.

24 Mino GABRIELE, Il viaggio dell'anima, in: A/G, S. IX.

25 GABRIELE, Il viaggio dell'anima, S. X.

26 Lefaivre, Leon Battista Alberti, S. 63, 65.

27 Ich schaute alsdann mit einzigartiger Wonne eine getäfelte Balkendecke, die, in höchstem Grade von Handwerk und Zier, als eine gleichmäßige Fläche aufgehängt war. Bar eines Gleichem oder Vergleichlichem, bildete sie einen stolzen Himmel; mit geordneten Zwischenraumeinteilungen von vielfältiger Gestaltung, welche mit errechneter und ebenmäßiger Bemessung [...] die Feldchen umsäumte; [...]

28 Zwischen jenen schaute ich wonniglich ein köstliches Tälchen, wo das wohlgefällige Grab meiner Seele lag $[\ldots]$ 
Cum extrema uoluptate contemplando questi nobilissimi concepti in tale figurato expressi mirai \& il quarto decontra al primo circulo. ${ }^{29}(H P, 245 ; 2$. Teil $)$

Liane Lefaivre deutet die Präsenz der voluptas in der Kunstbetrachtung als Erotisierung der Kunstobjekte und interpretiert alle Objekte und Gebäude als « embodiments of his beloved $»^{30}$ - eine auf den ersten Blick überzeugende These, die aber folgenden Umstand unterschlägt: Die Objekte und Gebäude werden nicht mit Attributen der Geliebten, sondern die Geliebte wird mit Attributen der Kunst beschrieben. Damit wäre es nicht die erotische voluptas, die überhandnimmt, sondern die ästhetische voluptas, die mehr und mehr Gewicht erhält und die erotische voluptas zurückdrängt.

Interessanterweise kommt keine der genannten Interpretationen auf das Staunen zu sprechen - obwohl es kaum eine Seite gibt, auf der es nicht explizit genannt wird. Diese Beobachtung widerspricht einer metaphysischen Deutung: Das Staunen richtet sich auf das, was da ist, weil die künstlerische Imagination es hervorgebracht hat. Der Leserblick wird gezielt von einer möglichen allegorischen Struktur und Interpretation auf den aisthetischästhetischen Eindruck gelenkt. Das Verfahren der $H P$ - so meine These - ist keines der metaphysischen Allegorisierung, sondern der Ästhetisierung, die mit Ausdrücken des Staunens einhergeht. Alle Objekte - von der Architektur über die Kunstwerke und Gärten bis hin zu Polia - werden staunend als Kunstobjekte betrachtet und beschrieben. ${ }^{31}$ Natur und Kunst gehen nicht ineinander auf, sondern die Natur wird ganz in den Bann der Kunst geschlagen. So ist in der Traumwelt der $H P$ nichts natürlicherweise da, sondern alle - organischen und anorganischen - Formen und Objekte wurden von einem erstaunlichen Künstleringenium geschaffen.

Dieser Vorzug der Kunstbetrachtung und -beschreibung gegenüber der Konstitution von Sinn lässt sich konkret beobachten, wenn sich eine allegorische Deutung an Stellen, die grosses allegorisches Potenzial böten, « im Luxus der konkreten Details » verflüchtigt. ${ }^{32}$ Das sehen wir beispielsweise in der Darstellung der vier Triumphwagen, die den Sieg Amors über Jupiter darstellen (Europa, Leda, Danae, Semele), dieser symbolische Gehalt aber durch die Beschreibung der Wagen und den darauf angebrachten Reliefs in den

29 Da ich mit äußerster Wonne diese allervornehmsten Gedanken, welche in solchem Bildschmuck ausgedrückt waren, in Augenschein nahm, schaute ich auch, der ersten Kreisfläche gegenüber, die vierte.

3 Lefaivre, Leon Battista Alberti, S. 3.

31 Vgl. Dickhaut, Positives Menschenbild, S. 173.

32 GoebeL, Erträumte Architektur, S. 49-51. 
Schatten gestellt wird. ${ }^{33}$ Zudem bietet der Text selbst eine explizite Auflösung symbolischer Darstellungen. So wird ausgeführt, dass die fünf Nymphen die fünf Sinne bedeuten oder dass Poliphilo sich im Reich einer Königin befindet, die den freien Willen symbolisiert. Im Reich dieser Königin trifft Poliphilo zudem auf die personifizierte Vernunft (Logistica), die er nach der Bedeutung der angetroffenen Objekte befragt und die ihm alles präzise und ausführlich erklärt $(H P, 132-141)$. Die Erklärung seiner Bedeutung zählt zur Analyse des Kunstwerkes wie das Eruieren materieller und technischer Beschaffenheit; sie wird offen dargelegt und es kommt ihr keinerlei übergeordnete Bedeutung zu.

Durch diese Ästhetisierung unterscheidet sich die HP markant von Vorgängertexten wie beispielsweise der Amorosa visione Boccaccios. Zeigen lässt sich dies an einem Beispiel: Sowohl Boccaccio als auch Colonna beschreiben einen Dreigrazien-Brunnen. Boccaccios Dreigrazien-Brunnen ist folgendermassen konzipiert (XXXVIII): Das Wasser entspringt einer schwarzbraunen Grazie aus den lachenden Augen, einer feuerroten Grazie aus der Brustwarze und einer weissen Grazie aus dem Kopf. ${ }^{34}$ Die drei Grazien stehen für drei unterschiedliche Formen der Liebe: die käufliche Liebe, die körperliche Liebe und die reine Liebe. ${ }^{35}$ Die $H P$ unterminiert eine allegorische Auslegung, indem sie alle drei Grazien gleich - gleiche Farbe, gleiche Haltung, gleiche Öffnung für das Wasser - präsentiert. Die drei goldenen Grazien stehen mit dem Rücken zueinander auf einem Sockel, das Wasser spritzt aus ihren Brustwarzen $(H P, 88-91)$.

Bei Colonna ist ein solcher [allegorischer] Sinn nicht erkennbar; dafür ist die Beschreibung viel reicher an plastischen Details, der architektonische Aufbau des Brunnens viel klarer als bei Boccaccio. ${ }^{36}$

Die Beschreibungen der $H P$ weisen ein Vielfaches an (technischen) Informationen über den Brunnen auf: die Breite des Beckens, das Material, dessen Dicke, die geometrische Form, die Verwendung von Elementen aus der

33 Ebd. Goebel spricht sich auch dafür aus, dass sich die $H P$ einer zahlensymbolischen Deutung widersetze (ebd., S. 59), während beispielsweise Silvia Fogliati quasi gegebenerweise davon ausgeht, dass die Zahlen symbolisch aufgelöst werden wollen und können. Siehe Silvia Fogliati, Lisola-giardino di Citèra, in: Fogliati / Dutto, Il giardino di Polifilo, S. 29-32.

34 Giovanni Boccaccio, Amorosa Visione, a cura di Vittore Branca, Milano 1974 (= Tutte le opere, vol. 3), Vv. $76-88$.

35 Zu Boccaccios innovativem Umgang mit der Allegorie siehe Johannes Bartuschat, L'ekphrasis dans la poésie allégorique médio-latine et dans l'« Amorosa Visione » de Boccace, in: Camenae 8 (2010), S. 2.

36 Goebel, Erträumte Architektur, S. 49. 
Mythologie, die Art der Säulen, die Verarbeitungsweise, die spezifische Ausprägung der unterschiedlichen Figuren, die kunstvolle Spring- und Auffangeinrichtung für das Wasser und nicht zuletzt die detaillierte Beschreibung der Grazien. ${ }^{37}$ Boccaccio lässt die Grazien von Personifizierungen der vier Kardinalstugenden umgeben sein, Poliphilo hingegen wählt vier Harpyien und vier « Drächlein » $(H P, 91)$, die insbesondere von dekorativem Wert sind $(H P, 89)$ und dem Brunnen gemeinsam mit den drei Grazien ein harmonischelegantes Gesamtbild verleihen. Die Beschreibungen der HP sind auf die künstlerische Konzeption und die Beschaffenheit des Brunnens gerichtet, die Brunnendarstellung der Amorosa visione zielt auf die Vermittlung eines allegorisch-moralischen Gehalts.

Schon Boccaccio hat seinen Protagonisten als träumenden Protagonisten konzipiert, dessen staunender Blick den Weg und die Erzählung gleichermassen leitet. $^{38}$

Oh quanto bella tal fonte pariami e quanto da lodar, tal che giammai di mirarla saziato non sariami, $[\ldots]^{39}$ (XXXIX, Vv. 1-3)

Rimirando n'andava intorno attento per lo gioioso loco, scalpitando l'erbette e' fiori col mio passo lento. Sì con diletto per il loco andando vidi in un verde e piccioletto prato una fonte di magister mirando. Io m'appressai a quella, e d'intagliato candido marmo vidi assai figure, ognuna in diversa aria ed atto e stato. Mirando quelle, vidi le scolture di diversi color, come compresi, qual belle e qual lucenti e quali oscure.

37 Im quantitativen Vergleich: Bei Boccaccio werden den Grazien zehn Verse gewidmet, in der $H P$ ist es eine gute Seite, bei der Brunnenbeschreibung insgesamt ist der Verhältnisunterschied noch beträchtlicher.

38 Vgl. dazu auch den Anfang des folgenden Sonnetts, das der Amorosa Visione vorangestellt ist: «Mirabil cosa forse la presente / vision vi parrà, donna gentile / a riguardar, sì per lo nuovo stile, / sì per la fantasia ch'è nella mente. (Eine erstaunliche Sache mag euch der gegenwärtige / Anblick, donna gentile, möglicherweise scheinen / zur Betrachtung, sowohl aufgrund des neuen Stils, / als auch wegen der Freiheit des Geistes.) » (1. Sonnett, Vv. 1-4, Übersetzung A.E.). Staunen, Bildlichkeit, neuer Stil und Fantasie werden hier einleitend genannt.

39 Oh, wie schön schien mir dieser Brunnen / und wie lobenswert, sodass ich nie / satt würde ihn zu bewundern. (Übersetzung A.E.) 
Vedeasi ivi un bel marmo; e quel sedesi

sovra la verde erbetta, di colore

porpureo tutto, e'n su quella stendesi

in piano e non di architettura fuore;

era in misura una canna per verso,

quadro ma basso e di vago splendore.

Sovra ogni angol di quel nitido e terso

di marmo una figura si sedea,

e ciascheduna aveva atto diverso,

ché più meravigliosa opra facea. ${ }^{40}$ (XXXVIII, Vv. 22-43)

Boccaccios Protagonist bestaunt die Kunstwerke: «una fonte di magister mirando » (V. 27), «vidi assai figure, / [...] / Mirando quelle, vidi le scolture» (Vv. 29-31), «meravigliosa opra facea » (V. 43). ${ }^{41}$ In der HP werden wir sehen, dass Poliphilo eine ebensolche staunend-betrachtende Haltung einnimmt, er aber die Kunstwerke als solche - in ihrer Beschaffenheit und Materialität - in den Vordergrund stellt, nicht deren (verborgene) Bedeutung.

\subsection{Begriffe}

Da ich in diesem Kapitel vermehrt zu spezifischen Begriffen wie „Ästhetik“, „ästhetische Betrachtung“, „Kunstwerk“, „Kunstbeschreibung“ und „Ekphrasis“ greife, erläutere ich deren Verwendungsweise an dieser Stelle.

Als „Ästhetik“ und „ästhetisch“ verstehe ich die sinnliche, insbesondere die visuelle Wahrnehmung von Kunstobjekten. Kunstobjekte und Kunstwerke sind Objekte, die nicht zu einem funktionalen Zweck, sondern als Ausdruck der menschlichen Imaginations- und Kreationskraft entstanden sind. Die Kunstwerke der $H P$ verkörpern Ausserordentlichkeit und imaginative Originalität auf der einen, die klassischen Kategorien von Harmonie, Ausgeglichenheit,

40 Aufmerksam schauend ging ich umher / an diesem freudigen Ort, auf die Gräser / und Blumen tretend mit meinem langsamen Gang. / Derart mit Vergnügen den Ort erkundend, / sah ich auf einer kleinen grünen Wiese / einen Brunnen von einem erstaunlichen Meister. / Ich näherte mich ihm und gemeisselt / in den weissen Marmor sah ich mehrere Figuren, / jede mit unterschiedlicher Miene, Handlung und Zustand. / Sie bestaunend sah ich die Skulpturen / in unterschiedlichen Farben, alles umfassend, / einige schön, einige strahlend, einige dunkel. / Dort sah man einen schönen Stein; sitzend / auf dem grünen Gras, ganz von purpurner / Farbe, liegend / in der Ebene und nicht ausserhalb der Struktur. / Er glich in der Grösse einem Gussrohr, / viereckig, aber tief und von anmutigem Glanz. / An jeder Ecke dieses klaren und reinen / Marmors sass eine Figur, / und jede hatte einen anderen Ausdruck, / was das Werk noch staunenswerter machte. (Übersetzung A.E.)

41 Zum Vergnügen der Kunstbetrachtung bei Boccaccio vgl. Johannes Bartuschat, Appunti sull'ecfrasi in Boccaccio, in: Italianistica 38 (2009), S. 71-9o. 
Ordnung und Schönheit auf der anderen Seite. Diese Qualitäten äussern sich in der Wirkung des Kunstwerkes, die sich insbesondere im Staunen manifestiert. $\mathrm{Zu}$ den Kunstobjekten zählen Werke der Architektur, der Bildhauerei, der Gartenkunst und der bildenden Kunst.

Beschreibungen der ästhetischen Wahrnehmung, vom Protagonisten in der ersten Person wiedergegeben, bilden das zentrale strukturelle Element der HP. Diese Beschreibungen beziehen sich nicht auf eine bestimmte Wirklichkeit, sondern sind «Präsentation[en] [...] ästhetischer Welten », die im und aus dem Traum "fingiert" werden. ${ }^{42}$ Ich bezeichne die Beschreibungen als Kunstbeschreibungen, Kunstdarstellungen oder Ekphrasen und behandle die drei Begriffe als Synonyme.

Den Begriff der Ekphrasis fasse ich relativ weit; ich meine damit sowohl « rhetorische Übung », « literarische Technik » als auch eine Form der Erkenntnis bzw. Erkenntnisgewinnung, wobei alle drei Ausprägungen in der $H P$ stets auf das Kunstwerk bezogen bleiben und nicht für sprachliche Anschaulichkeit im Allgemeinen stehen. ${ }^{43}$

Poliphilos Kunstbeschreibungen sind ein Spiel des Nach- mit dem Auf- und Nebeneinander - das Nacheinander ist die Funktionsweise der Sprache, das Auf- und Nebeneinander jene des Bildes bzw. der Skulptur. Die Beschreibungen schildern die Schichten, vollziehen sich aber im Nacheinander der Sprache und sind eingebettet in eine fortlaufende Rahmenhandlung. ${ }^{44}$ Das immer detailliertere Beschreiben ist massgeblich vom Staunen geleitet, aus dem das Interesse am Betrachten, Erkennen und Beschreiben des Gesehenen überhaupt erst hervorgeht.

Die Ekphrasis der $H P$ fusst wie schon die Ekphrasis der lateinischen und der mittelalterlichen Literatur auf dem Spannungsfeld zwischen «illusion»

42 Gottfried Boehm und Helmut Pfotenhauer, Einleitung: Wege der Beschreibung, in: DiEs. (Hgg.), Beschreibungskunst - Kunstbeschreibung. Ekphrasis von der Antike bis zur Gegenwart, München 1995, S. 9. In den Einleitungen der $H P$ heisst es explizit, es werde berichtet, was Poliphilo im Traum gesehen und erfunden habe $(H P, 5)$.

43 Boemi / Pfotenhauer, Einleitung, S. 10.

44 Vgl. dazu den ausführlichen Artikel von Giovanni Pozzi, Il «Polifilo» nella storia del libro illustrato veneziano, in: De Rs., Sull'orlo del visibile parlare, Milano 1993, S. 89-113. Spannend wäre es zudem, das in der $H P$ angewandte Verfahren mit Panofskys Aufsatz Zum Problem der Beschreibung und Inhaltsdeutung von Werken der bildenden Kunst (1932) zu untersuchen, denn Panofsky wählt als optimale Strategie für die Bildbeschreibung ein «schrittweises Verfahren, in dem sich das Bild in die verschiedenen Schichten zerlegt » und neben der Beschreibung insbesondere auch die Einstellung des Betrachters ins Feld geführt wird. Das Zitat staummt aus Gottfried Boenm, Bildbeschreibung. Über die Grenzen von Bild und Sprache, in: Boeнm / PFOтеnHAUer, Beschreibungskunst - Kunstbeschreibung, S. 30-31. 
und « instrument de connaissance $», 45$ wobei « connaissance» im Falle der $H P$ nicht auf Wissen im Allgemeinen zielt - also nicht auf Wissen über den Menschen, Gott, das Universum, die Tugend o. Ä. -, sondern auf das Verständnis des Kunstwerkes und dessen kreativer Entstehung. Insbesondere die moralische Perspektive hat in der $H P$ wenig Gewicht. Bezeugt wird die Fähigkeit des Künstlers, Bilder, Skulpturen und Gebäude zu schaffen, die in ihrer Vollkommenheit den "Werken“ der Natur und vor allem der antiken Künstler in nichts nachstehen. Es geht also insbesondere darum, wie etwas konzipiert und kreiert worden ist, woraus es besteht, wie es wirkt, und weniger darum, was dargestellt wird. Damit befindet sich die Ekphrasis in der HP nahe an der Schwelle zur sachlichen Beschreibung (descriptio), wird aber stets in die subjektive und affektive Wahrnehmung des Protagonisten zurückgeholt. Aus dem Blickwinkel des Staunens heraus betrachtet heisst das: Die « illusion » - Imagination und Umsetzung des Künstlers - erzeugt Staunen. Das Staunen regt zur genauen Betrachtung und Beschreibung an, wobei mehr «connaissance» nicht zu weniger Staunen, sondern im Gegenteil zu noch grösserer Bewunderung (des Werkes und des Künstlers) führt.

Dass Kunstbeschreibungen nicht mehr in erster Linie der Illustration oder dem Kommentar der Handlung dienen, bezeichnen Boehm und Pfotenhauer als Errungenschaft der Moderne. Diese modernen Kunstbeschreibungen repräsentieren «uneinlösbare Imagination und selbstreflexive Gestaltungsverfahren $»{ }^{46}$ Eine entsprechende Tendenz lässt sich auch für die Kunstbeschreibungen der HP erkennen. Doch während die Bilder der Moderne und der Gegenwart sich von der « Benennbarkeit durch die Sprache, von verbindlichen Referenzen » entfernen, wird die «Bildkraft der Sprache» in der $H P$ vorgeführt und bestärkt. ${ }^{47}$ Es gibt somit keinen Bruch zwischen Sprache und Bild, ${ }^{48}$ sondern das Bestreben, Bild und Sprache zusammenzuführen und durch die genaue Beschreibung ihre Funktionsweisen - explizit für die Kunst, implizit für die Literatur - zu ergründen. Auch im Zeigecharakter der Ekphrasis manifestiert sich eine Konvergenz der «bildgebende[n] Leistung der Sprache mit der ursprünglichen Leistung des Bildes», wobei in der $H P$ der zeigende Gestus ein staunender ist und das Dargestellte entsprechend von erstaunlichem Charakter zeugt. ${ }^{49}$

45 Bartuschat, L'ekphrasis, S. 4.

46 Boenm / Pfotenhauer, Einleitung, S. 9.

47 Ebd., S. 10.

48 Von einer «Sprachskepsis » in Bezug auf das Bild, wie wir es heute kennen, kann keine Rede sein, auch wenn verschiedentlich der Unsagbarkeitstopos verwendet wird. Dieser hat in erster Linie die Funktion, das Aussergewöhnliche des zu beschreibenden Objektes herauszuheben. Siehe dazu Bоєнм, Bildbeschreibung, S. 23.

BоЕнм, Bildbeschreibung, S. 35 . 
Die detaillierte Aufzählung aller Eigenschaften gehört traditionellerweise zur Ekphrasis. Dabei ist diese Aufzählung nicht eine einfache Akkumulation, sondern stellt ein organisches Ganzes dar. ${ }^{50}$ Die Wahrnehmung des organischen Ganzen manifestiert sich insbesondere in den Bezeugungen der Wirkung. In der $H P$ wird dieser globale Eindruck stets vom Staunen begleitet: Die Ekphrasen starten mit dem Staunen, münden in eine Detailbeschreibung und werden durch eine erneute Staunensbekundung abgerundet. Sie sagen, «was, ist'» und zugleich auch, «wie es, wirkt"», was auch als «Gefälle zwischen Fakten und Affekten » bezeichnet werden kann. ${ }^{51}$ Für die $H P$ werden wir sehen, dass sich Affekte und Fakten nicht gegenüberstehen, sondern durch das Staunen miteinander verschränkt sind. ${ }^{52}$

\subsection{Das Staunen als visuelle Wahrnehmung}

Candide Poliphilum narrantem somnia lector

Auscultes, summo somnia missa Polo,

Non operam perdes, non hæc audisse pigebit.

Tam uariis mirum rebus abundat opus.

Si grauis \& tetricus contemnis erotica, rerum

Nosce precor seriem tam bene dispositam.

Abnuis? ac saltem stylus \& noua lingua nouusque

Sermo grauis, sophia serogat aspicias. ${ }^{53}(H P, 3, \mathrm{Vv} .19-25)$

50 BARtuschat, L'ekphrasis, S. 6: « [...] un ensemble organique, soutenu par l'idée de l'ordre et de la totalité.»

51 Bовнм, Bildbeschreibung, S. 3 о.

$5^{2}$ Der staunenden Beschreibung eines Kunstwerkes werden wir einige Jahrzehnte später bei Vasari wiederbegegnen. Auch er bettet die «Form- und Kompositionsanalyse» in die staunende Wahrnehmung des erzählenden Betrachters ein. Entgegen früheren Annahmen, die Kunstbeschreibungen würden in den Vite nur eine anekdotische « Nebenrolle spielen», ist man sich heute einig darüber, dass Vasari ein ganzes «Spektrum rhetorischer Wirkungsqualitäten » - insbesondere auch des Staunens - innerhalb einer «Verschränkung verschiedener Textsorten » entfaltet, in der die Kunstbeschreibung keine Neben- sondern eine Hauptrolle spielt. So müsste auch Vasari auf das Staunen hin untersucht werden, während die $H P$ Eingang finden sollte in die Studien zur Ekphrasis, in denen sie bisher quasi unerwähnt geblieben ist. Siehe dazu Воєнм, Bildbeschreibung, S. 31-33. Für eine Analyse der Ekphrasis in Vasaris Werken vgl. Svetlana AlPERs, Ekphrasis und Kunstanschauung in Vasaris Viten, in: Boenm/Pfotenhauer, Beschreibungskunst Kunstbeschreibung, S. 217-258. Ebenfalls im selben Band: Matthias Winner, Ekphrasis bei Vasari, S. 259-278.

53 Lausche, geneigtester Leser, Poliphilo, da er Träume / Erzählt, welche vom Himmel gesandt worden sind, / Nicht ist's Mühen vergeblich, auch nicht wird's Hören gar Dich reu'n, / Denn vor solch Mannigfalt geht über das staunliche Werk. / Wenn ernst Du wie auch finster verachtest Erotik, erkenn' der / Dinge Abfolge an, ich bitte, solch planvoll gefügt. / Nein sagst Du? Dann ziemt's sich zumindest, dem Stil wie der neuen / Sprache 
Eine anonyme Elegie, die dem Werk vorangestellt ist, preist die Vorzüge der $H P$ : Die Träume, die darin erzählt werden, seien himmlischen Ursprungs. Die Handlung des Werkes sei hervorragend komponiert. Stil und Sprache würden sich in einem neuen Gewand präsentieren. Dem Auge und dem Geist würden mannigfaltige Bilder mit unerwartetem, lehrreichem Inhalt geboten.

In einer ausführlichen Nacherzählung des Inhalts wird der Leser darauf vorbereitet, was er im Laufe der Lektüre wird betrachten können: « darnach lese » («hinc lege», V. 21), « erschaue darnach» («inde [...] specta», V. 24), «bald $[\ldots]$ sichtest $»(« \operatorname{mox}[\ldots]$ cernes , Vv. 31-32), «hier schau» («hic [...] uide», V. 37), « hier kannst Du auch beäugen » («hicque [...] poteris spectare», V. 39). Dieser Gestus des „Da wirst du sehen“, „Schau hier!“ nimmt die Eigenheit der $H P$, mit Sprache detaillierte Bildwelten entstehen zu lassen, vorweg.

Welche Bilder das sind, wird in der zweiten Einleitung detailliert ausgeführt:

Lector si tu desideri intendere breuemente quello che in questopera se contiene, Sapi che Poliphilo narra hauere in sogno uisto mirande cose, laquale opera ello per uocabulo græco la chiama pugna damor in sogno. Oue lui finge auere uisto molte cose antiquarie digne di memoria, \& tutto quello lui dice hauere uisto di puncto in puncto \& per proprii uocabuli ello descriue cum elegante stilo, Pyramide, obelisci, Ruine maxime di edificii. La differentia di columne la sua mensura, gli capitelli, base, epistyli, cioe trabi recti, trabi inflexi, zophori, cioe frisii, coronice cum gli sui ornati. Vno magno caballo. Vno maximo elephanto, Vno colosso, una porta magnifica, cum le mensure \& gli sui ornamenti, uno spauento, li cinque sentimenti in cinque nymphe uno egregio bagno, fontane, el palatio della regina che e el libero arbitrio. uno regio pasto \& superexcellente. La uarietate di zoie ouero petre preziose \& la sua natura. Vno gioco de scachi in ballo atre mensure de soni. Tre giardini, uno di uitro, uno di seta, uno in laberyntho che e la uita umana $[\ldots]^{54}(H P, 5)$

und würd'gem Ausdruck, Weisheit doch Achtung zu zoll'n. // Das Zitat stammt aus der Elegie eines Unbekannten (Anonymi elegia Ad Lectorem; HP, 3), die sich in der ersten Ausgabe von 1499 dem Romantext vorangestellt findet - sie wird sowohl von Ariani als auch von Pozzi dem Autor der HP zugeschrieben. Siehe dazu Reiser, Kommentar, S. 6.

54 Leser, wenn Du erwünschst, in kürze dasjenige zu vernehmen, welches in diesesm Werke enthalten ist, wisse, daß Poliphilo erzählt, er habe im Träume staunliche Dinge gesehen. Jenes Werk nennt er mit griechischem Begriff, Kampf um Liebe im Traume'. Darin dichtet er, er habe viele altertümliche, der Erinnerung würdige Dinge gesehen. Und all dasjenige, von dem er sagt, es gesehen zu haben, beschreibt er auf das genaueste mit den gehörigen Begriffen in elegentam Stil: Pyramiden, Obelisken, gewaltige Ruinen von Bauwerken, die Verschiedenheit der Säulen, ihre Bemessung, die Kapitelle, Basen, Epistylia, das heißt gerades Gebälk, eingebogenes Gebälk, Zophoroi, das heißt Friese, Kranzgesimse mit deren Profilierung; ein großes Pferd, einen gewaltigen Elefanten, einen Koloß, ein prächtiges Portal, mit seinen Bemessungen und seinen Verzierungen, ein Schreckbild, die fünf Sinne als Nymphen, ein ausgezeichnetes Bad, Brunnen, den Palast als Königin, welche das ausgewogene Urteil darstellt; einen königlichen und sehr herausragenden Festschmaus; die Mannigfalt der Juwelen oder aber kostbaren Steine und deren Natur; 
Poliphilo erfindet («finge») in der Erzählung die erstaunlichen Figuren, Gebäude und Orte («mirande cose»), die er im Traum gesehen hat, damit sie für die Augen der Leser zugänglich werden. Die Vorwegnahme quasi aller Traumstationen zeigt deutlich, dass diese nicht als Bestandteile der Handlung aussergewöhnlich sind, sondern dass die Art und Weise, wie Poliphilo sie erfindet und beschreibt - mit eigenen Worten und einem eleganten Stil -, von besonderem Interesse sein wird. Die Dramatik des narrativen Strangs Poliphilo, der von seiner Geliebten Polia abgewiesen wird und sie dann im Traum wiederfindet - wird einleitend hingegen nicht erwähnt.

Werden die Leser vom Text zum Staunen angeleitet, ist es für Poliphilo Polia, die ihn zum Betrachten und Staunen führt:

Polia el conduce amirare quatro triumphi mirandi di Ioue, le amorosi deli Dei. $(H P, 5)^{55}$

Nel quale Polia suade a Poliphilo el uadi intro a mirare le cose antiche. $(H P, 5)^{56}$

Gleichzeitig zählt Polia zu den betrachteten Kunstwerken; sie wird als «Kunstwerk» («artificio», $H P, 239-240$ ), als gemaltes Werk («picturato», «colorato ») oder Schauspiel («placidissimo spectaculo », HP, 287), am Ende des zweiten Buches als «staunliches Abbild » («miranda imagine », HP, 463) bezeichnet. ${ }^{57}$

Das Gesehene wird in hyperbolische Ausdrücke des Staunens eingebettet. So zum Beispiel in der Beschreibung einer «ALLererlesenste [N] Pforte » $(H P, 30)$ der Pyramide. ${ }^{58}$ Die Portaltür ist derart perfekt geformt, dass sie der Beschreibung würdig ist:

ein nach drei Taktmaßen getanztes Schachspiel; drei Gärten, einer von Glas, einer von Seide, einer als Labyrinth, welches das menschliche Leben darstellt; [...]

55 Polia geleitet ihn, vier staunliche Triumphe Jupiters zu schauen, die Liebschaften der Götter [...]

$5^{6}$ In welchen Polia Poliphilo hineinzugehen überzeugt, um die altertümlichen Dinge zu schauen.

57 Vgl. dazu beispielsweise den Beginn des Romans, wenn Polia als «effigie angelica » bezeichnet wird $(H P, 11)$. Die Stilisierung zu einem Bild bleibt konstant und im Vordergrund, abwechselnd werden ihr zudem Attribute fiktiver Frauenfiguren (Jungfrau Maria, Venus, Beatrice) zugeschrieben. Sie wird als Bild komponiert, das sich aus diesen VorBildern zusammensetzt. Erst im zweiten Buch brechen realistische Züge in ihre Figur hinein, wobei auch hier - entgegen Gerhard Goebel, der das zweite Buch der "Wirklichkeit" zuordnet - eine starke Idealisierung der Figur zu erkennen ist. Siehe GoEbEL, Erträumte Architektur, S. 63.

$5^{8}$ Besonders augenfällig ist die Stelle $H P, 57-58$. 
Digno per tanto horaio exstimo il perfecto suo componimento descriuere. ${ }^{59}$ $(H P, 47)$

Die architektonische Komposition wird folgendermassen aufgefächert:

1. Ein Werk von «ausgesuchter Bauordnung und Kunst», das «wunderbarlich zusammengefügt war», wird mit Staunen betrachtet und damit ins Blickfeld des Lesers gerückt. $(H P, 42)$

2. Poliphilos «Begierde entflammt» und der Intellekt setzt ein, um das «scharfe Vorausdenken [...] des Architekten », dessen «Berechnungen » im «Entwurf» und in der «Ausführung» $\mathrm{zu}$ verstehen. $(H P, 42)$ Die gesamte Beschreibung wird immer wieder durchbrochen von Äusserungen der Bewunderung gegenüber dem Architekten und seinem Erfindungsvermögen. $\left(H P, 5^{\mathrm{O}-51}\right)$

3. Poliphilo beginnt, die Tür zu vermessen, und erhofft sich, daraus auf das Kompositionsprinzip des Architekten schliessen zu können. Die technische Vermessung wird begleitet von einer Analyse der materiellen Beschaffenheit.

Der Torbogen zeugt von erhabener, waghalsiger Fiktionskraft und eleganter Oberflächenglättung $\left(H P, 5^{\circ}\right)$. Auf der Frontseite der Portaltür ist der Raub des Ganimedes in «äußerst allerschwärzeste[n]» Stein gemeisselt. Poliphilo nimmt dieses Detail „erstarrt“ («mirai attonito », HP, 50) zur Kenntnis.

Mirai attonito dunque in una pugnace \& nigerrima petra Auqila paulo meno che tutta euulsa dal solido cum le ale aperte. Laquale hauea amorosamente rapto uno ingenuo \& delicatissimo Puello per gli sui panniculi. [...] Per il quale exquisitissimo expresso isteti stupefacto excogitando. Como lo elegante artifice cusi perspicacemente se imaginoe di applicare aptissimamente quella petra ad tale officio \& proposito. ${ }^{60}\left(H P, 5^{\mathrm{O}}-51\right)$

Das erstaunte Nachdenken («isteti stupefacto excogitando ») betrifft nicht den mythologischen Inhalt, der allgemein bekannt ist, ebenso wenig richtet

59 Als würdig erachte ich es deswegen jetzt, seine vollkommene Zusammenfügung zu beschreiben.

6o Bestürzt schaute ich da nun einen Adler, der mit geöffneten Flügeln aus einem wehrhaften und äußerst allerschwärzestem Stein, etwas weniger als ganz aus dem Massiven, herausgewölbt war. Jener hatte aus Verliebtheit einen wohlgeborenen und äußerst anziehenden Jungen an dessen Lumpen gepackt; [...] Ob jener allererlesensten Ausführung verblüfft geriet ich in Nachsinnen darüber, wie der elegante Künstler es sich also scharfsinnig vorgestellt hatte, jenen Stein in vollkommener Weise zu solchem Zwecke und Vorhaben zu verwenden. 
es sich - wie bei Platon und Aristoteles - auf das Metaphysische. Erstaunlich sind einzig und allein die Neuartigkeit und die Genialität der künstlerischen Umsetzung.

Die visuelle Anziehungskraft von Bildern und Skulpturen ist grösser als die lebendiger Figuren: So gleitet der Blick des Protagonisten weg von den Nymphen, die ihn auf seinem Weg begleiten, hin zu den Säulen, die künstliche Nymphen darstellen, und weiter zum Mosaik des Fussbodens. Dem staunenden Blick («miraua», «uedeuasi ») entspricht die wunderbare Qualität des Betrachteten («merauegliosi graphidi »):

O quanto exquisitiamente sculpte miraua le dicte imagine, che piu delle fiate, gli occhii mei dalle uere \& reale deuiare concedeua \& riportarliad le fincte.

La pauitata areola sotto laqua di uaria emblematura di petre dure tessellate, in merauegliosi graphidi per diuersi coloramenti uedeuasi. ${ }^{61}(H P, 82)$

Die Thermen insgesamt, zu denen die Säulen und der Mosaikboden gehören, regen zum Staunen an:

Di quanti mai gli antiquarii inuentori in duritudine fingere præstamente ualseno. Opera dædalea \& di admiratione conspicua. ${ }^{62}(H P, 89)$

«[D]i admiratione conspicua» wird als Qualität des dädalischen Werkes dargestellt.

Das bewundernde Betrachten steigert sich bisweilen zu einem Staunen, das die Sinne ausser Kraft setzt ( «stupore insensato », HP, 25) oder zu einem erstarrten Staunen («mirai attonito », $H P, 158)$ anwächst.

Auf der Insel Kythera gelangen Polia und Poliphilo «an den allerannehmlichsten Ort [...], welcher sich [...] dermaßen entzückend und schön,

61 Oh, so sehr ausgesucht gehauen schaute ich die besagten Bildnisse, daß ich mehrere Male meinen Augen zugestand, von den wahren und tatsächlichen abzuschweifen und sie zurückzuführen auf die Nachgebildeten. Man sah die Fußbodenfläche unter Wasser von mannigen Einlegearbeiten, aus harten Steinen gewürfelt, in wunderbaren Zeichnungen von verschiedenem Farbenspiele.

$62[\ldots]$ wie sie nie die altertümlichen Erfinder in hartem Material auf vortreffliche Weise zu bilden vermocht hatten; ein Daedalisches Werk zum Erstaunen schön anzusehen. // Der letzte Teilsatz des Zitats ist nicht eindeutig und nur schwer übersetzbar - ich würde « conspicua di admiratione » als „von Verwunderung in die Augen fallend“ lesen, Reiser übersetzt mit «zum Erstaunen schön anzusehen». Genau genommen ist «di admiratione » kein Adverb, wie Reiser dies übersetzt, sondern eine kausale Präzisierung des Adjektivs « conspicuo »: Die Kunstobjekte haben eine augenfällige Qualität, weil sie voller Bewunderung (Bewunderungswürdigkeit) sind. Weitere Beispiele finden sich in den Abschnitten $H P, 25$ und $H P, 93$. 
von einzigartigem Baumschmuck, den Sinnen darbot, wie man nie etwas Herausragenderes oder Sinnlicheres mit den Augen schauen könnte» (HP, 292). Die Reaktion auf die Gärten der Insel fällt unterschiedlich stark aus - mal unbeschwert vergnüglich, mal erstarrend. ${ }^{63}$ Wahrgenommen werden durchwegs ästhetische Eigenschaften: von der « eximia dispositione» («vortreffliche Anordnung») hin zu Eleganz, Harmonie und Vielfalt $(H P, 357)$. Wie schon Polia und die Nymphen wird auch der Garten nicht als Natur, sondern nur als künstlerisch geformter zum Objekt von Poliphilos Blick.

Wir haben gesehen, wie sich auf « Kythera» der locus amoenus ins Künstliche steigert: von den Hainen der Peripherie über die opera topiaria des zweiten und dritten Ringes zur steinernen Architektur der bugartigen Mitte. Architektur aber, ob grüne oder steinerne, ist die ganze Insel $[\ldots]^{64}$

Die genannte ars topiaria ist krönender Ausdruck der Artifizialität der Insel; unter anderem ragen die in Sträucher geschnittenen herkuleischen Taten heraus $(H P, 295) \cdot{ }^{65}$

Künstlich ist aber nicht nur die Natur - das Gesehene im Allgemeinen -, sondern auch die Qualität des Blickes selbst. Dies zeigt sich beispielsweise in der « anomale[n] Schärfe des Blicks », die von « vollkommener Rücksichtslosigkeit gegenüber perzeptivem Realismus [zeugt] - und [...] eben darum vielleicht umso besser der Realität des Traumes entspricht ».66 Teil dieses onirischen Realismus ist der «perspektivische[] Irrealismus » von Poliphilos Blick, der beispielsweise die Insel Kythera aus der Vogelperspektive beschreibt. ${ }^{67}$

Keine Eigenschaft von Poliphilos Blick, sondern eine spezifische Wirkung der fiktiven Welt auf sein Auge ist der Trompe-l'CEil-Effekt. An verschiedenen Stellen werden Natürlichkeit und Lebendigkeit vorgetäuscht: Ein Eingangszimmer im Palast der Eleuterilyda ist mit Pflanzenhecken und Himmelsgewölben bemalt, was den Eindruck eines echten Gartens erweckt $(H P, 94){ }^{68}$ Im Garten des Palastes entdeckt Poliphilo einen Brunnen mit einem Mosaik, das Fische darstellt $(H P, 82)$. In der Reflexion des Lichtes scheinen diese Fische

63 Vgl. $H P, 293,295,298,306,308,324,357$.

64 Goebel, Erträumte Architektur, S. 59. Vgl. dazu ebenso LefaIVre, Leon Battista Alberti, S. 52 .

65 Fogliati, L'isola di Citèra, S. 28.

66 Goebel, Erträumte Architektur, S. 54.

67 Ebd., S. 6o.

68 Lefaivre, Leon Battista Alberti, S. 53. 
sich lebendig zu bewegen. Im Tempel der Venus wird ein Raum beschrieben, dessen Wände von sich bewegenden Bildern animiert werden $(H P, 208) \cdot{ }^{69}$ Die Artifizialität der Bilder wird dabei immer herausgestellt.

Es zeigt sich also, dass auf der einen Seite das Auge Poliphilos in dieser literarischen Traumwelt Fähigkeiten entwickelt, die ihm im realen Leben verwehrt blieben. Auf der anderen Seite weist das Gesehene ausserordentliche und täuschende (fingierende) Eigenschaften auf, die das Auge auf eine spezielle, artifizielle Art und Weise ansprechen.

Die Betrachtung der Kunstwerke wird mit Verben beschrieben, die das Staunen und die Bewunderung in die visuelle Wahrnehmung einschliessen: Sehen und Staunen erweisen sich als zusammengehörig. ${ }^{70} \mathrm{Zu}$ diesen Verben zählen (re)mirare ${ }^{71}$, spectare ${ }^{72}$ und videre, wobei letzteres stets mit einem Adverb oder Objekt des Staunens ergänzt wird:

Douete sapere chio uidi la mirauegliosa fontana $[\ldots]^{73}(H P, 79)$

Cum decorata disseminatione di multiplice \& inextimabile gemme mirificamente uidi cum ornato. ${ }^{74}(H P, 95)$

Vidi due historiale digne di maxima admiratione in tale scalptura. Nella facia dinanti di esso uaso mirai incisura optimamente lo altitonante Ioue. ${ }^{75}(H P, 174)$

Nel proximio latere, uidi de miranda celatura, una Damigella nel aspecto uirgineo $[\ldots]^{76}(H P, 193)$

69 Lefaivre, Leon Battista Alberti, S. 56-57.

70 Vergleichbare Stellen finden sich auf folgenden Seiten: $H P, 61,72,80,92,159,171,173,190$, 196, 221, 236, 239, 242, 243, 244, 248, 250, 257, 260, 272, 299, 346, 372.

71 Lat. mïror: 1. sich wundern, sich verwundern, 2. etw. od. jmd. bewundernd anstaunen, nach etw. od. jmd. mit Bewunderung hinblicken, etw. od. jmd. bewundernd verehren. Siehe Der neue Georges.

72 Lat. specto: schauen = sehen, anschauen, ansehen, betrachten, I. 1. Sehen, mit ansehen, ihm zusehen, beiwohnen, 2. a bewundernd anschauen [...] b prüfend betrachten, prüfen, untersuchen $[\ldots]$ (ebd.)

73 Ihr sollt wissen, daß ich die wunderbarliche Quelle sah.

74 Die restliche ebene Fläche sah ich [...] in verschönter Verstreuung auf wundersame Weise mit vielartigen und unersinnlichen Juwelen geziert.

$75[\ldots]$ sah ich zwei, der größten Bewunderung würdige Historiendarstellungen von der folgenden Bildhauerarbeit: Auf der Vorderfront des nämlichen Gefäßes schaute [ich] allerbestens in Steinschnitt eingeschnitten den Hochdonnernden Jupiter.

${ }_{76}$ Auf der nächsten Seite sah ich von staunlicher Reliefarbeit ein Fräulein von jungfräulichem Ansehen [...] 
Fora dillaquale, purissimo fumo uidi miraculosamente uscire germinando, \& successiuamente multiplicantise in uno uerdigiante rosario. ${ }^{77}(H P, 233)$

Das Staunen wird als Konsequenz des genauen Betrachtens bezeichnet:

Da conuertire in stupore chi acuratissimo, \& cum obstinato intuito la consideraua. ${ }^{78}(H P, 24)$

Folgendes lässt sich schliessen: Die Verben des Sehens und Betrachtens weisen eine spezifisch ästhetische Konnotation auf und sind an das Staunen gekoppelt.

\subsubsection{Die Kapitelzusammenfassungen}

Für jedes der 38 Kapitel bietet der Autor kurze, einleitende Kapitelzusammenfassungen, die sich in drei Teile gliedern: ${ }^{79}$ Die Zusammenfassungen I-X verweisen auf die Erzählungen Poliphilos, wie er im Traumreich aufwacht und eine Landschaft durchwandert, die durchsetzt ist mit ausserordentlichen (Kunst-) Objekten. Die Zusammenfassungen XI-XXIV kündigen Berichte aus dem Reich der Liebe an, in dem sich Poliphilo und Polia begegnen und sie ihre Liebe mit verschiedenen Riten initiieren und bekräftigen. Der dritte Teil Kapitel XXV-XXXVIII - bildet das zweite Buch der HP, in dem die beiden Liebenden abwechslungsweise und retrospektiv die Anfänge ihrer Liebesgeschichte entfalten. ${ }^{80}$

\subsubsection{1. Teil (I-X)}

Nach zwei einleitenden Kapiteln markiert das dritte Kapitel den eigentlichen Einstieg in das Traumreich des Romans - Poliphilo ist im Traum eingeschlafen und beginnt erneut zu träumen.

77 Aus demselben sah ich allerreinsten Dampf sprießend herauskommen, der sich nach und nach zu einem grünenden Rosenstrauch vervielfältigte [...]

78 Es [das Kunstwerk, in diesem Fall ein Obelisk] war im Stande denjenigen in Verwunderung zu stürzen, welcher sie auf das genauste und mit hartnäckigem Blick in Augenschein nahm.

79 Die Gliederung basiert auf einer von mir vorgenommenen Einteilung. Sie ist motiviert zum einen durch die Handlung, zum anderen durch eine unterschiedliche Rolle des Staunens in den drei Teilen.

8o Während der erste Teil sich aus 130 und der letzte aus gut 8o Seiten zusammensetzt, beläuft sich der mittlere Teil auf 240 Seiten und ist damit deutlich länger als die beiden anderen Teile. Es könnte auf den ersten Blick scheinen, dass das Treffen der beiden Liebenden wichtiger sei als die Kunsterzählungen. Aber weil auch der zweite Teil zu einem Grossteil aus Kunst- und Architekturbeschreibungen besteht, ist dieser erste Eindruck zu relativieren. 
poliphilo qvivi narra, che gli parve ancora di dormire, et altronde in somno ritrovarse in vna convalle, la qvale nel fine era serata de vna mirabile clavsvra cvm vna portentosa pyramide, de admiratione digna, et vno excelso obelisco de sopra. la quale cvm diligentia et piacere svbtilmente la consideroe. ${ }^{81}(H P, 20)$

Poliphilo erzählt ausschliesslich von erstaunlichen Dingen («MIRABILE CLAVSVRA », «VNA PORTENTOSA PYRAMIDE, DE ADMIRATIONE DIGNE », «VNO EXCELSO OBELISCO ») und Vorkommnissen. So nehmen auch die folgenden Kapitelzusammenfassungen vorweg, dass Poliphilo von einem «STAUNENSWERTEN OBELISKEN» («MiRANDO OBELISCO», HP, 3O), einer grossen Portaltür mit kunstfertigem («FABERRIMO»), erstaunlichem («MIRO») Portalschmuck $\left(H P, 47,5^{8}\right)$ und einem wunderbar gefertigten Brunnen («VNA EXIMIA OPERA DI FONANTA», HP, 79) berichten wird. Weiter wird er von der Begegnung mit fünf Nymphen erzählen, die über seine Erscheinung verwundert sind («MERAVIGLIANTISE », HP, 68). Diese Nymphen führen ihn zum Palast einer Königin, wobei Poliphilo und die Königin sich gegenseitig bestaunen werden $(H P, 94)$. In diesem Palast wird ein wunderbares Festmahl («mirabile [...] conuito ») veranstaltet, die Nymphen zeigen Poliphilo exquisite Dinge, damit er diese bewundern kann (« el conduseron admirare [...]», HP, 117). Zum Schluss führen sie Poliphilo zu drei Türen, von denen Poliphilo die mittlere, die ins Reich der Liebe führt, wählt.

\subsubsection{2. Teil (XI-XXIV)}

Poliphilo bewegt sich nicht mehr allein durch das Traumreich, sondern wird von Polia durch dieses geführt. Sie zeigt ihm die Triumphzüge zu Ehren Jupiters; Tempel, in denen Liebesriten durchgeführt werden; antike Inschriften; die Insel Kythera mit ihren Gärten; den Brunnen der Venus und das Amphitheater. Im Gegensatz zum ersten Teil, in dem die Sehnsucht nach Polia nur sporadisch aufflammte und die Erregung von neckischen und reizvollen Nymphen hervorgerufen wurde, wird die Liebeserregung in der Anwesenheit Polias zum ständigen Begleiter. Nur durch die ruhige Betrachtung der Kunstwerke oder der «STAUnenswerten Schönheiten» («Mirabile [...] Bellezza», $H P, 153)$ der Geliebten kann Poliphilo seine verzehrende Erregung lindern («SACQVietoe [...] Nel SVO DOLCE ASPECTO MIRANDo », HP, 181).

81 Poliphilo erzählt allhier, dass es ihm schien, noch zu schlafen und im Traum sich anderswo zu befinden: in einem Talkessel, welcher am Ende von einem staunenswerten Damm mit einer phantastischen Pyramide verschlossen war, würdig der Bewunderung, und einem aufragenden Obelisken obenauf. Jene nahm er feinsinnig mit Aufmerksamkeit und Wohlgefallen in Augenschein. 
Wie schon im ersten Teil stehen auch im zweiten Teil vorwiegend antike Bauten und Kunstwerke im Vordergrund. Allerdings wird die Schönheit Polias $(H P, 224)$ zu einem Motiv, das die Kunstbetrachtungen von nun an begleitet.

\subsubsection{3. Teil (XXV-XXXVIII)}

In den letzten vierzehn Kapiteln des Buches wird Polia von ihrer Herkunft sowie von ihren ersten Begegnungen mit Poliphilo berichten, bevor auch Poliphilo rückblickend erzählen wird, wo er Polia zum ersten Mal gesehen und sich in sie verliebt hat. In diesem Teil spielen Kunst- und Architekturobjekte kaum eine Rolle.

Aus der Analyse der Kapiteleinführungen können wir feststellen, dass das erste Buch der HP der Betrachtung von Kunst, Architektur und Gärten gewidmet ist, während das Betrachten im zweiten Buch durch das retrospektive Erzählen abgelöst wird. Die angekündigten Objekte des ersten Buches werden mit wiederholten, ja geradezu exzessiv benutzten Ausdrücken des Staunens bedacht, während diese im zweiten Buch kaum mehr eine Rolle spielen. Das erste und das zweite Buch unterscheiden sich auch im Fiktionsgrad voneinander. Das erste Buch spielt in einer imaginären Welt, das zweite Buch verfährt « wirklichkeitsähnlich », 82 quasi als Übergang zur Welt ausserhalb des Traums. Das erste Buch besteht vorwiegend aus der Ekphrasis einer fantasievollen Traumwelt, im zweiten Buch hingegen stehen die handelnden und erzählenden Figuren - Polia und Poliphilo - im Vordergrund.

\subsubsection{Die Traumreise}

\subsubsection{1. Teil}

Wie schon die Kapitelresümees ankündigen, ist der erste Teil des Buches voll mit Beschreibungen von Werken mit erstaunlichen Qualitäten. Diese Beschreibungen bestimmen auch massgeblich die Struktur der Kapitel: Poliphilo befindet sich zuerst in einem « FINSTEREN WALD » $(H P, 11)$, dann in einem lieblichen Tal. Er bewegt sich durch diese seltsamen Gegenden und erblickt immer wieder erstaunliche Dinge, ${ }^{83}$ beispielsweise die Statuen eines

82 Rainer Stillers, Die Verwandlung der Bilder in Worte. Erzählte Poetik in Boccaccios Amorosa visione, in: Heidi Marek, Anne Neuschäfer und Susanne Tichy (Hgg.), Metamorphosen. Wandlungen und Verwandlungen in Literatur, Sprache und Kunst von der Antike bis zur Gegenwart, Festschrift für Bodo Guthmüller zum 65. Geburtstag, Wiesbaden 2002, S. 27.

83 Für den Leser ist die Fortbewegung Poliphilos nicht immer linear nachvollziehbar. Teilweise scheint es eher, Poliphilo - und mit ihm der Leser - stehe still und die Objekte zögen vor seinen Augen vorüber. Die Bilder erhalten damit einen sequenziellen Charakter, wie er sonst Texten eigen ist. 
Pferdes, eines Kolosses ${ }^{84}$ und eines Elefanten. ${ }^{85}$ Vor diesen bleibt er stehen, bewundert, studiert und beschreibt sie - anfänglich gepaart mit Furcht und Unsicherheit, ${ }^{86}$ zunehmend mit mehr und mehr Vergnügen. ${ }^{87}$

Beim Pferd staunt Poliphilo darüber, dass die «machina » sich in ein Pferd gewandelt hat: Die Natürlichkeit der Kunst steht im Vordergrund, Maschine und Natur verschmelzen miteinander als Resultat des menschlichen Ingeniums. Die Kunst löst die Natur in ihrem schöpferischen Vermögen ab:

Stupefacto dunque non poco, rumindando, \& cum summo dilecto curioso riguardando tale ingente machina conflata in animale da humano ingenio, dignissimo imaginato. ${ }^{88}(H P, 35)$

So ist es auch die Kunst, genauer ein Koloss, der die Anatomie des Menschen in erstaunlicher Manier vorführt:

Echo chio uedo uno Vastissimo \& mirando colosso [...] Ilquale iaceua decumbendo supino di metallo mirabile artificio conflato, di media ætate, subleuato alquanto sopra uno puluino tenendo il capo. Cum sembiante di ægro, cum la bucca di suspirare \& gemere indicante. hiante, di preceritate passi 6o. Et per li crini sopra il pecto se poteua ascendere, Et per li tomentati \& tormentati pili dilla fulta barba, alla lamentabonda bucca. Il quale meatosamente era tutto iane \& uacuo. Per quella dunque dal curioso scrutario stimulo, senza altro consultamine impulso, nella gula per graduli introgresso \& dindi nel stomaco, Et dequi cum latebrosi ducti ad tutte laltre parte delle interne uiscere, alquanto pauoritato perueni, o mirando concepto, io mirai tutte le parte intimamente, quale in uno humano corpo peruie. ${ }^{89}\left(H P, 35^{-36}\right)$

84 Interessanterweise gibt es vom Koloss keine Illustration - im Gegensatz zu anderen beschriebenen Figuren -, die $H P$ verweist die Leser auf ihre eigene Imagination. Siehe LefaIVRe, Leon Battista Alberti, S. 55.

85 Vgl. dazu Fogliati, Hypnerotomachia Poliphili, S. 13. Ebenfalls dazu die Illustrationen in der $H P$ auf den Seiten $26,32,38,55$.

$86 H P, 13$.

$87 \quad H P, 22$.

88 Da ich nun nicht wenig erstaunt, geistig durchkaute und mit höchstem Vergnügen neugierig betrachtete einen, vom menschlichen Geiste zu solch Tiergestalt zusammengeschmolzenen, gewaltigen Geniestreich [...]

$89[\ldots]$ da sehe ich einen sich äußerst weit erstreckenden und staunenswerten Koloß [...] Jener lag darniederliegend rücklings; eine aus Metall gegossene erstaunliche Kunstarbeit; von mittlerem Alter, angehoben ein wenig das auf einem Kissen gehaltene Haupt; mit dem Erscheinungsbild des Kranken, mit von Seufzen und Stöhnen klaffendem Munde; von der Erstreckung 6o Schritt. Sowohl über die Haare auf der Brust als auch über die gewundenen und gedrehten Haare des dichten Bartes konnte man hinaufsteigen zum jammernden Mund. Jener war in der Art eines Ganges ganz leer und hohl. Durch jenen stieg ich nun, vom neugierigen Forscherdrang gedrängt, ohne weitere Überlegungen anzustellen. Als ich über kleine Stufen in den Schlund [36] hineingeschritten war, 
Der Koloss ist riesig (« uastissimo ») und erstaunlich («mirando »). Es wird ausführlich beschrieben, wie er aussieht, wie gross er ist und was das Spezielle an ihm ist - nämlich, dass er einen kranken Menschen darstellt, in den man - «oh staunlicher Entwurf» («oh mirando concepto ») - hineinsteigen und alle Teile des Körpers durchwandern und betrachten kann. Die Idee und die Konzeption des Kolosses werden besonders hervorgehoben. Der antike Künstler schafft es, mittels herausragender inventio und ars die Anatomie des Menschen unmittelbar und prägnant vor Augen zu führen $(H P, 36)$.

Auch der Elefant stellt ein zu Architektur gewordenes Lebewesen dar. Er ist aus schwarzem Edelstein geformt und trägt einen Obelisken auf seinem Sattel. Wie in den Koloss steigt Poliphilo in die «Elefanten-Konstruktion» hinein, denn sie verfügt über einen begehbaren Hohlraum $(H P, 38)$. Im Innern des Elefanten kann Poliphilo sich aufrecht bewegen. Im hinteren und im vorderen Teil des Elefanten befinden sich Sarkophage, in die seltsame Inschriften eingraviert sind. Poliphilo ist verblüfft, wobei die Verblüffung von einem Schaudern begleitet ist: «Ob jener ungewöhnlichen Sache, war ich mit einigem Schrecken nicht mäßig verblüfft. $(H P, 39)^{90}$ Das Neue und Unheimliche ist einen Bericht wert (« digna di relato »):

Di tanta nouitate digna di relato mirabondo, \& degli ænigmati prælegendoli sæpicule, dil tutto io restai ignaro, \& dilla interpretatione \& sosphismo significato molto ambiguo..$^{91}(H P, 40)$

Poliphilo kann die ambivalente Bedeutung nicht entschlüsseln und versucht sie auch nicht weiter zu ergründen. Das Innere des Elefanten offenbart nicht ein Wissen, wie es das Körperinnere des Kolosses tat, sondern enigmatische Bilder und Texte, die sich einer eindeutigen Interpretation widersetzen. Wir sehen uns somit mit zwei unterschiedlichen Arten erstaunlicher Objekte konfrontiert: Zuerst wird den Sinnen unmittelbares Wissen offenbart, dann wird das Rätselhafte, das dem Wissen nicht zugänglich ist, betont.

Erst später, als Poliphilo von Logistica und Telemia zu den drei Toren des freien Willens geführt wird, wird Poliphilo über die rätselhaften Sarkophage

dann von dort in den Magen. Und von diesem gelangte ich, ein wenig beklommen, über verschlupfwinkelte Gänge zu all den anderen Teilen der inneren Eingeweide. Oh staunlicher Entwurf! Ich schaute alle Teile im Inneren, so wie in einem menschlichen Körper durchgängig.

90 Per laquale inusitata cosa istetti non mediocremente stupido cum alquanto horrore. // Lat. horror: 1. das Zusammenfahren, der Schauer [...], 2. a der Schauder, Grausen, Entsetzen, b der heilige Schauer, c der Wonneschauer. Siehe Der neue Georges.

91 Da ich über solch Ungeschautes und Rätselhaftes, wert des Berichts, staunte, blieb ich, obschon ich sie ziemlich oft vorlas, im ganzen unkundig wie auch sehr im Zweifel bezüglich der Auslegung und der von Sophismen bedeckten Bedeutung. 
im Elefanten Auskunft erhalten. Allerdings wird ihm nicht deren Bedeutung offenbart, sondern die geniale Konstruktion vor Augen gehalten:

Poliphile so pienamente quello che inquiri, Vorei pero che tu sapesti, che non senza grande admiratione di humano ingegno \& cum ardente studio \& incredibile diligentia fue fabricata quella ingente machina, Cum perplexibilitate dello intellecto ad intendere il suo diuino concepto. ${ }^{92}(H P, 132)$

Die herausragenden Werke dienen als Verweis auf die grossartige, gottähnliche Schöpfungskraft des Menschen. Sie zeugen von leidenschaftlicher Arbeit («ardente studio») und unglaublicher Sorgfalt («incredibile diligentia»). Logistica gibt denn auch keine umfassenden Erklärungen ab, sondern nur Hinweise, auf deren Basis Poliphilo - und ebenso der Leser - weiterdenken soll: «Gehe dies jetzt in Deinem Sinn gründlich durch!» $(H P, 134)$

Hätte der «Naturgeschichtler» (Plinius) alle diese Werke schon gekannt, hätte er sie als die höchsten aller Kunstwerke beschrieben - höher als das "wunderbarliche Kunstwerk des vornehmen Memnon », als die Werke des Architekten Satyrus, als das « erstaunliche Wunder der in den Berg Bagistanus gehauenen Statue der großherzigen Semiramis » $(H P, 58-59) \cdot{ }^{93}$ Die $H P$ führt in diesen Passagen eine Steigerung des Staunens über den Vergleich durch: Die in der $H P$ beschriebenen Werke werden superlativ mit Werken verglichen, die bisher in der Literatur, der Geschichte und der Mythologie als erstaunlichste galten. Überboten wird dies zusätzlich dadurch, dass Poliphilo den Werken in einem ruinösen Zustand begegnet - es sind antike Werke, viele sind nicht mehr vollständig erhalten, in der Witterung zerfallen oder von Pflanzen überwuchert. Wenn selbst diese Werke Poliphilo in einen Zustand «erstaunlicher Bewunderung» und «Ergötzen» $(H P, 59)$ versetzen, wie staunenerregend wären dann die vollkommenen Werke?

Si gli fragmenti dilla sancta antiquitate \& rupture \& ruinamento \& quodammodo le Scobe ne ducono in stupenda admiratione, \& ad tanto oblectamento di mirarle, quanto farebbe la sua integritate ${ }^{94}(H P, 59)$

92 Poliphilo, ich weiß völlig, wessen Du nachforscht. Ich möchte freilich, daß Du wissest, daß jene gewaltige Konstruktion nicht ohne großes Augenmerk des menschlichen Geistes und mit loderndem Eifer und unglaublicher Umsicht gefertigt ward, um beim Verstehen ihres göttlichen Entwurfes für die Verwirrung des Verstandes zu sorgen.

93 Eine Liste der Werke, die gemäss dem Erzähler von den erstaunlichen Werken in der HP übertroffen werden, findet sich auf folgenden Seiten: $H P,{ }_{5}^{8-} 59$.

94 Wenn die Trümmer des verehrungswürdigen Altertums und Bruchstücke und Einstürze, gewissermaßen die Sägespäne davon, in uns zu solch erstaunlicher Bewunderung und so Ergötzen, sie zu schauen, verleiten, wie viel bewirkte ihre Unversehrtheit? 
Nach dem Eintritt in das Portal betrachtet Poliphilo erst staunend mit offenem Mund («cum gli labri aperti », HP, 61) die Gemälde und Skulpturen im Innern, wird dann aber von einem Drachen erschreckt und in die Enge getrieben. Nur durch Glück findet er einen Ausgang in die liebliche Landschaft auf der anderen Seite der Pyramide. Als Poliphilo den ersten Schrecken überwunden hat, denkt er wehmütig an all die Werke, die seine Sinne mit Freude erfüllt haben, zurück.

Et in momento dal profundo dil tristo core trahendo gli gemitosi sospiri, nella tenace reminiscentia replicaua quanto piacere \& dilecto in puncto haueano gli sensi mei perdito, Imperoche quella operatura era piena di merauiglia, \& di stupore. $(H P, 67)^{95}$

Nach diesem kurzen Innehalten erblickt Poliphilo ein « achteckiges Gebäude » mit einem wunderbaren Brunnen («mirabile \& egregia fontana», HP, 70), wobei ihn eine Nymphenstatue besonders fasziniert. Diese ist von derart perfekter Schönheit und lebendiger Erscheinung, dass sie eine sexuelle Wirkung auf ihre Betrachter ausübt $(H P, 71)$. Im Vordergrund steht das Staunen darüber, dass der Künstler mit seinem Kunstwerk eine solche Wirkung zu erzeugen vermochte. Mit dem Verstand kann Poliphilo sich solch eine Leistung kaum erklären und behilft sich der Vorstellung einer Petrifikation.

Et quanto uenustamente bellissima lui la expresse, tanto chegli homini insacrilega concupiscentia di quella exarsi, il simulachro masturbando stuprorono. ${ }^{96} \mathrm{Ma}$ quanto ualeua æstimare dritamente arbitrai tale imagine mai fusse cusi perfecta di celte, ouero di scalpello simulata, che quasi ragioneuolmente suspicaui, in questo loco de uiua essere lapidita \& cusi petrificata. ${ }^{97}(H P, 71)$

Die Nymphen, denen er kurz darauf begegnet, führen ihn zu einem achteckigen Gebäude: den Thermen. Er entdeckt Reliefs, Gemälde, Mosaike und

95 Und da ich augenblicklich die stöhnenden Seufzer aus der Tiefe des traurigen Herzens zog, wiederholte sich in der hartnäckigen Wiedererinnerung genau, wie viel an Gefallen und Entzücken meine Sinne verloren hatten, aus dem Grunde, daß jenes Bauwerk voll von Verwunderung war und von Staunen.

96 Der Autor schafft an dieser Stelle ein Wortspiel zwischen stupor und stupro, wobei stupro in diesem Fall nicht (nur) negativ konnotiert ist, sondern im Gegenteil die unglaubliche Wirkung der Statue betont.

97 Und in dermaßen reizender Weise wunderschön hatte er sie ausgeführt, daß die Männer, in weihebrüchigem Gelüste nach jener entbrannt, das Götterbild masturbierend schändeten. Soweit ich es aber einzuschätzen vermochte, kam ich geradewegs zu dem Urteil, daß ein solches Abbild niemals also vollkommen mit dem Meißel ausgeführt sein könnte oder mit dem Eisen nachgebildet, so, daß ich gleichsam billig vermutete, daß es lebendig an diesem Orte zu Fels geworden und also versteinert wäre. 
beschreibt diese detailliert auf mehreren Seiten $(H P, 80-85)$. Allerdings lenken die Nymphen ihn von seinen Betrachtungen ab und laden ihn ein, gemeinsam mit ihnen ein Bad zu nehmen. Sie reichen ihm eine Salbe, die ihn nicht - wie von diesen behauptet - belebt, sondern ihn in einem « wolllüstige[n] Lodern » $(H P, 87)$ animalisch und schmerzhaft erregt. Das unkontrollierbare Ausgeliefertsein an körperliche Begehren wird als qualvoll beschrieben.

Angekommen im Palast der Eleuterilyda - der Königin des freien Willens -, wird die Handlung durch entzückte Ausrufe sistiert:

Quale marmori, quale scalpture, Oue mirai le uirtute Herculane in petra luculea Simidiuulse mirabilmente exscalpte. ${ }^{98}(H P, 93)$

Die Skulpturen sind «auf staunenswerte Weise ausgehauen », auf «wunderbare Weise gemeißelt», die Decke ist «wunderbar» und «wunderschön kassettiert», die Elemente sind « königlich », « erlesen[] » und « elegant», der ganze Palast ist ein « staunliches Gebäude » $(H P, 93)$. Auf dem Weg zur Königin begegnet Poliphilo zahlreichen weiteren Kunstwerken: Gemälden, Skulpturen und Teppichen - um nur einige zu nennen -, die ausführlich beschrieben werden. Auch die Königin und die Mädchen - insbesondere deren Thron, Gewänder, Schmuck - werden zu Objekten einer ausführlichen Ekphrasis (HP, 99, ebenso 102-116).

Schliesslich fordert die erstaunte Königin Poliphilo auf, ihr zu berichten, wie er den Drachen hat überwinden und in ihr Reich gelangen können $(H P, 101)$. Poliphilo steht verblüfft und demütig vor der Königin und ihren Damen. Er ist kein stolzer Held, sondern ein bescheidener, zufällig in den Palast geratener (Kunst-)Liebhaber. Kurz, aber ausführlich $(H P, 101)$ beschreibt er seine Flucht vor dem Drachen, bald aber richtet sich sein ganzes Begehren («summa uoluptate ») wieder auf die Pracht des Palastes $(H P, 101)$.

Die Szene im Palast führt deutlich vor Augen, dass Poliphilos Rolle jene des Betrachters ist. Er nimmt am Geschehen teil wie an einer «Darbietung» $(H P$, 110): Er betrachtet, staunt, denkt, beschreibt - und greift nur widerwillig als agierendes Subjekt in die Handlung ein.

Auf ein festliches Mahl folgt ein Schachspiel, das mit lebenden und tanzenden Figuren gespielt wird, und nach all diesen « allerherausragendsten und wunderbaren Dinge[n]» («præexcellentissime \& mirauegliose cose», $H P, 123)$ bleiben vier Werke zu bewundern: ein Garten aus Glas - «von größter Einfallsgabe» («mirabile fincto», $H P, 124)$-, ein labyrinthischer

98 Welch Marmor! Welch Bildhauerkunst, wo ich schaute die Herkulischen Arbeiten in Lucullischen Stein, halb losgelöst auf staunenswerte Weise ausgehauen! 
Garten-eine «staunliche[] Anlage » (« mirandosito »,HP,125)-, ein Garten aus Seide und Juwelen - «von staunlicher Kunstarbeit» («mirando opificio», $H P, 127)$ - sowie ein Tempel mit Arkaden, der unter anderem die Dreifaltigkeit symbolisiert und Poliphilo mit mehr Freude erfüllt als alle vorausgegangenen Wunderwerke («che qualunque altra mirabile opera », $H P, 131$ ).

Schliesslich öffnet Poliphilo das Tor in das Reich der Liebe; ${ }^{99}$ es beginnt der zweite Teil.

\subsubsection{2. Teil}

Kaum geht er durch die Tür, wird Poliphilo von lasziven Mädchen umgarnt und schliesslich allein zurückgelassen. Wie schon nach der Episode mit dem Drachen, als Poliphilo sich allein auf der anderen Seite der Pyramide wiederfand, denkt er auch an dieser Stelle an die « cose stupende transacte » zurück.

EXCESSIVAMENTE IL MiO TENERO core damorose punctiture percosso, non intendo si io deliraua che cusi rimansi stupefacto, in che modo da gliocchii mei, \& cusi repente il gratissimo consortio euanescente disparue. [...] Sotto di questa intrando grauemente anxio circa la inopinata priuatione, \& ricogitabondo delle uarie \& magne \& cose stupende transacte. ${ }^{100}(H P, 141)$

Poliphilo weiss nicht, ob er deliriert oder ob er richtig gesehen hat, so sehr ist er vom plötzlichen Verschwinden seiner Begleiterinnen erstaunt («cusi rimansi stupefacto »). Das Staunen schafft so immer auch Brüche und Übergänge, die das Ende des einen und den Beginn des nächsten Teils markieren - dargestellt anhand des Passierens in ein neues (Traum-)Reich mittels einer Tür oder eines Durchgangs.

Der Moment der Erinnerung währt nur kurz, bald schon erblickt Poliphilo eine Gruppe tanzender Mädchen. Aus dieser Gruppe löst sich eine Nymphe ${ }^{101}$ von derart herausragender Schönheit und Eleganz, dass selbst die schönste aller Göttinnen vor ihr erblasst wäre. Wie schon in Polizianos Stanze markiert

99 Poliphilo kann zwischen drei Türen wählen: theodoxia (gloria di dio), cosmodoxia (gloria del mondo) und erototrophos (madre d'amore, $H P, 135$ ).

$100[. .$.$] mein zartes Herz im Übermaße so von Liebesstichen durchbohrt - ganz so, als$ ob ich Wahnvorstellungen hätte, verblüfft zurückblieb, nicht, auf welche Weise die allerwillkommenste Gesellschaft aus meinen Augen und, indem sie sich also schalgartig auflöste, verschwand. [...] Als ich, ob der unvermuteten Beraubung schwer beklommen, unter jene eintrat und wieder der mannigen und großen wie auch erstaunlichen durchgemachten Dinge gedachte [...]

101 Es handelt sich um Polia, die sich Poliphilo aber erst später zu erkennen gibt. Poliphilo erkennt zwar die Ähnlichkeit zu Polia, ist sich aber nicht sicher, ob es sich tatsächlich um sie handelt, da ihre Erscheinung als Nymphe ungewohnt und fremd auf ihn wirkt. 
eine Nymphe als traumhaftes Wesen mit gleichzeitig antik-mythischen und historischen Zügen die entscheidende Wendung.

Poliphilo beschreibt die Nymphe als «Abbild» («repræsentatione», $H P$, 147) und drückt Verblüffung («remansi stupido \& arrepto », «verharrte ich [...] verblüfft und ergriffen ») vor ihrem Bild («dolce effigie », « süße[s] Ebenbild », $H P, 148$ ), sinnliche und emotionale Verwunderung («sentiui gli spiriti mei stupefacti», «[d]anun gewahrte ich, wie meine Geister wie gebannt waren », $H P, 148$; «miro affecto», «staunliche[] Leidenschaft», $H P, 153$ ) und Bewunderung aus («tutto diuerecunda admiratione reimpleto», «von Ehrfurcht und Bewunderung angefüllt», $H P$, 149). Er würde fortfahren, sie zu betrachten, würde diese seinen Blick nicht von sich weg und hin auf die «staunlichen und heiligen Stätten» («mirauegliosi \& sancti loci») und die «staunlichen Triumphzüge » («mirandi triumphi », $H P, 154)$ lenken. Poliphilos Blick kehrt dennoch immer wieder zur Nymphe zurück.

[...] miarchitatrice propinquo essendo obstupefacto miraua che contra omni naturale ordine, quella me piu morbidamente inficiaua. ${ }^{102}(H P, 242)$

Später wird Poliphilo sich gar wünschen, die wunderschönen Nymphen seien Statuen anstatt lebendiger Körper $(H P, 337)$, denn als solche wären sie nicht imstande, sein schmerzhaftes Liebesbegehren heraufzubeschwören. Als Ausweg bleibt Poliphilo die Beschreibung der Nymphen, als wären sie Statuen $(H P, 342)$ - durch die literarische Transformation in Kunstwerke bezwingt Poliphilo die quälende Erregung. Damit erinnert Poliphilo an Pygmalion, der sich sein gewünschtes Frauenbild als Statue erschuf, in das er sich verliebte und das Venus schliesslich lebendig werden liess. Pygmalion und Poliphilo schaffen sich in der literarisch-bildlichen Traumwelt eine Liebesutopie, wobei Pygmalion das Künstliche lebendig, Poliphilo hingegen das Lebendige künstlich werden lässt.

Es folgt die Beschreibung eines Tempels, die ihren Höhepunkt in einer rauschenden accumulatio findet:

de diuo excogitato, \& de superba operatura, \& mirandi liniamenti, de stupenda ostentatione, condito mirabilissimo. ${ }^{103}(H P, 214)$

$102[\ldots]$ denn obwohl ich sah, daß ich mich nahe bei dem [...] Heilmittel und bei meiner Leibärztin befand, nahm ich doch verblüfft war, daß jene mir wider jede natürliche Regel noch mehr Siechtum zufügte.

$103[\ldots]$ von göttlicher Ersinnung und von stolzer Ausführung und staunlichen Profilierungen, von verblüffender Stattlichkeit, allerwunderbarster Anordnung [...] 
Die Grossartigkeit des Tempels offenbart die göttliche Idee, die exzellente Ausführung, die bewundernswerte Linienführung, die verblüffende Zurschaustellung, die wunderbarste aller Bauweisen.

Erst an dieser Stelle gibt Polia sich zu erkennen und kündigt einen Opferund Liebesritus an, der mit der Priesterin des Tempels vollzogen werde. Zwischen die Ankündigung des Opferritus und dessen Vollzug wird abermals eine ausführliche Ekphrasis (der Kapelle) eingefügt und die Spannung der Liebeshandlung gleichermassen aufgebrochen.104

Erst dann folgt der Ritus, den Poliphilo in einer Mischung aus angsterfülltem Staunen, Befremden und Faszination verfolgt:

Per laquale cosa io stupefacto \& totalmente alienato, \& timido tuto effecto, intanto cosiche in capo capillo non ristoe, che subleuato non fusse, cum lanimo molto suspeso, dubitando pensiculatamente in questo solenne \& sacro piamento non fusse surrepta la mia ingenua Polia [...] Ma cum suspectosa admiratione peruicacemente obseruaua notando quello aptissima faceua lei \& inseme la summa Antistite. ${ }^{105}(H P, 227-228)$

Onde pieno \& circumfacto di miraueglioso terrore, \& paurosamente agitato, inuocaua silentioso, qualunque diuino subsidio \& pietate. Et apena alquanto aperti gli spauentati occhii riguardai alla fumante ara. Fora dillaquale, purissimo fumo uidi miracolosamente uscire germinando, \& successiuamente multiplicantise in uno uerdigiante rosario. ${ }^{106}(H P, 233)$

Das Zusammenkommen von Staunen und Furcht findet sich in jenen Momenten, in denen sich Poliphilo in der fremden Umgebung unsicher oder ängstlich fühlt und in denen er um Polia fürchtet (vgl. $H P$, 100). Ebenso wird

104 Die Kapelle ist « auf staunliche Weise errichtet » aus kostbarem Stein. Dieser Stein ist von « wundervoller Beschaffenheit», denn er beleuchtet den Innenraum der Kapelle, ohne dass Licht über Fenster in diesen eindringt $(H P, 219)$. Auch die weiteren Details der Kapelle weisen wunderbare Qualitäten auf, so die «mira urna hyacinthina» («staunliche[] hyazinthene[] Urnula ») oder der « mirando candelabro aureo » («staunliche[] güld'ne[] Kandelaber », $H P$, 225).

105 Ob jener Sache war ich dermaßen verblüfft und gänzlich befremdet und ganz eingeschüchtert, daß auf dem Haupt kein Haar verblieb, welches nicht zu Berge gestanden wäre; mit recht argwöhnischem Sinn, grüblerisch bezweifelnd, ob mir bei diesem feierlichen und heiligen Sühneopfer nicht meine wohlgeborene Polia entrückt würde [...] [228] mit argwöhnischer Bewunderung beobachtete ich aufmerkend dasjenige, welches sie allerbefähigst machte; wie auch gemeinsam mit der Vorsteherin.

106 Darob von wundersamen Schrecken erfüllt und umwirbelt und furchtbarlich aufgebracht, rief ich still jedweden göttlichen Beistand und Erbarmen an. Und kaum hatte ich ein wenig die entsetzten Augen aufgetan, schaute ich hin zum dampfenden Altare. Aus demselben sah ich allerreinsten Dampf sprießend herauskommen, der sich nach und nach zu einem grünenden Rosenstrauch vervielfältigte [...] 
Furcht in Zusammenhang mit Wundern - an dieser Stelle das Spriessen und Wachsen des Rosenkranzes - genannt. ${ }^{107}$

Durch den Ritus erfährt Poliphilo eine «neuartige[...] Beschaffenheit der Liebe », die es ihm ermöglichen, die « venerischen Gnaden » zu erkennen $(H P$, 234). Die Verwandlung bleibt rätselhaft und wird nicht genauer ausgeführt. Zudem zeigt sie weder auf Polia noch auf Poliphilo einen sichtbaren Einfluss, denn dieser fährt nach dem Ritus in gewohnter Manier fort, Polia und die Traumlandschaft zu betrachten. Schliesslich wird er bald wieder von seinen «Liebesmartern » $(H P, 242)$ eingeholt, von denen ihn Polia - wiederum nach bekanntem Muster - ablenkt. Kurz vor der Überfahrt nach Kythera schickt sie Poliphilo zu den Tempelruinen und leitet damit aufs Neue eine Passage ausführlicher Kunstbetrachtungen ein. Das erotische Begehren wird transformiert in eine obsessive Haltung des Staunens gegenüber den Kunstwerken:

mirai uno obelisco ${ }^{108}(H P, 243)$

mirai ancora tali eleganti hieroglyphi ${ }^{109}(H P, 244)$

mirai tale designature di egregio expresso ${ }^{110}(H P, 245)$

Cum extrema uoluptate contemplabondo questi nobilisimi concepti in tale figurato expressi mirai $[\ldots]^{111}$ (Ebd.)

Per laquale cancellatura mirando $[\ldots]^{112}(H P, 247)$

Tute queste cose cum grande \& smisurato piacere, \& singular deuotione diligentemente mirate di sopra ritornai. Oue mirabondo dilla integritate di questa insigne operatura di ciborio. ${ }^{113}(H P, 248)$

107 Furcht zeigt sich auch zu Beginn, als Poliphilo sich in der selva obscura befindet, die ihm unheimlich erscheint, und er von wilden Tieren umgeben ist; vor dem Drachen, der ihn in der Pyramide bedroht; als er im Palast der Eleuterilyda von den jungen Damen bestürmt wird, ohne dass er weiss, was mit ihm geschieht; als er die Tempelruine besichtigt und plötzlich erschrocken vor Angst um Polia an den Strand zurückkehrt; oder als er am Ende des ersten Buches vor der Statue der Venus Staunen und Schrecken $(H P, 362)$ empfindet, weil er fürchtet, das gleiche Schicksal wie Aristeus zu erleiden, der die nackte Diana beim Baden erblickte und daraufhin von dieser in einen Hirsch verwandelt wurde.

$108[\ldots]$ schaute ich $[\ldots]$ einen $[\ldots]$ Obelisken $[\ldots]$

$109[\ldots]$ schaute ich nun zwei weitere elegante Hierglyphen $[\ldots]$

110 [...] schaute ich die folgende Darstellung von ausgezeichnetem Ausdruck [...]

111 Da ich mit äußerster Wonne diese allervornehmsten Gedanken, welche in solchem Bildschmuck ausgedrückt waren, in Augenschein nahm, [...]

112 Als ich durch jenes Gitterwerk schaute, [...]

113 Nachdem ich all diese Dinge mit großem und maßlosem Vergnügen und einzigartiger Andacht sorgfältig geschaut hatte, kehrte ich nach oben zurück. Dort bekräftigte ich, über die Unversehrtheit dieser ausgezeichneten Arbeit von Gitterwerk staunend, [...] 
Finalmente cum tale dispositione mirai questo odioso, spauenteuole \& euitando Barathro $[\ldots]^{114}(H P, 250)$

Oue picto mirai uno homo affligente una damigella. ${ }^{115}(H P, 257)$

Cum maxima delectatione \& piacere questi spectandi fragmenti mirando, auido piu anchora indagante altro di nouo trouare. ${ }^{116}(H P, 260)$

Nella quale mirai impressa una facie di regia maiestate coronata cum barba prolixa, \& la cæsarie intorta. ${ }^{117}(H P, 265)$

Facile non mi se præsta cum quanta hylaritudine io accuratissimamente miraua tanto di memorato uenerande opere opportunamente narrare. ${ }^{118}(H P, 268)$

Dique io solamente miraua parte de uno fluuiolo, pareua uestigio di humana forma in quello tramutata, di arte incredibile fincto, \& mirabilmente expresso. Quale simigliante unque nel delubro di Minerua in capitolio nella tabula se uide il rapto dilla dicta, da Nicomacho depicto. ${ }^{119}(H P, 272)$

Auf der Überfahrt nach Kythera erleben wir einen kurzen Exkurs in die Welt der Musik: Polia singt gemeinsam mit den «schmuckesten Ruderinnen» himmlische Melodien - so sehr zur Bewunderung der Zuhörer («in admiratione prouocando »), wie es nicht einmal Demodoch mit seiner « Leier» $\mathrm{zu}$ erreichen vermochte $(H P, 289)$. Ähnlich wird es auf der Insel Kythera sein, wenn die Nymphen mit «wunderbarlichen wie auch mit geblasenen Instrumenten » («marauegliosi, \& cum organati instrumenti », HP, 343) zu spielen beginnen oder im Amphitheater ein Stimmenkonzert von tanzenden Nymphen zu hören ist, das den Gesetzen des «Gleichmaß[es| des Taktes der Mildheit und der Harmonie » $(H P, 356)$ entspricht. Das auditive Element ist im

114 Letztlich schaute ich in solcher Ausführung diesen häßlichen, entsetzlichen und zu meidenden Höllenschlund, [...]

115 Dort schaute ich einen Mann abgebildet, der ein Fräulein züchtigt [...]

116 Da ich mit größtem Ergötzen und Vergnügen diese ansehlichen Bruchstücke schaute, forschte ich noch neugieriger nach, $[\ldots]$

117 In jenem schaute ich eingegraben ein Gesicht von königlicher Hoheit, gekrönt; mit breitem Barte, das kurze Haar eingedreht.

118 Leicht stellte es sich nicht für mich dar, angemessen wiederzugeben, mit wie großer Heiterkeit ich auf das Sorgfältigste dermaßen der Erinnerung würdige Kunstwerke schaute.

119 Daher schaute ich alleiniglich einen Teil des Flüßchens, es schien die Spur einer, in jenes umgewandelten menschlichen Gestalt zu sein; von unglaublicher Kunst verfertigt und in staunlicher Weise ausgeführt. Solchem ähnlich schien niemals im stillen Wohnsitz der Minerva auf dem Kapitol der von Nikomachos auf der Bildtafel abgeschilderte Raub der Besagten. 
Gegensatz zum stark betonten visuellen Moment nur selten präsent; es folgt dem platonischen Ideal der himmlischen Harmonie und wird mehr mit Wohlgefallen als mit Staunen in Verbindung gebracht.

Auf der Insel Kythera wird die Gartenanlage zum Objekt des Staunens. Alle Elemente - seien dies Baumreihen, aus Büschen geformte Szenen der Mythologie, kunstvoll gefertigte Brunnen, Mosaike, Peristyle und Epistyle oder Treppenaufgänge - zeugen von quasi übernatürlicher Imaginations- und Gestaltungskraft:

Cum tutti gli difertati miraculi attonito \& exanimo io totalmente restai. ${ }^{120}$ $(H P, 357)$

Höhepunkt der Insel ist das zentral gelegene antike (Amphi-)Theater. Es übertrifft alle berühmten antiken Theater und wird als Anlage, die einst Austragungsort von Spektakeln war, selbst zum staunenswerten Spektakel: ${ }^{121}$

Io nel primo aspecto, uno miraculo molto et grandemente stupendo uidi. ${ }^{122}$ $\left(H P, 35^{2}\right)$

Die einzelnen Reihen im Theater sind von Pflanzen verschiedenster Arten bewachsen und werden botanisch detailgetreu beschrieben. Poliphilo ist von der « Hoheit des Ortes » so sehr eingenommen, dass sogar seine Liebe zu Polia verblasst und seine « inwärtigen wie auch auswärtigen Sinne » ausser sich sind $(H P, 357)$. In der Mitte des Theaters befindet sich das «WUNDERbarliche Kunstwerk des Venerischen Quells» $(H P, 358)$ der Venus. Auch für diese werden Bewunderung und Staunen zu fast schon tautologischen Beschreibungselementen - neben Eigenschaften wie Neuheit und Exzellenz.

In questo loco, at etiam di questo ammirando fonte la nouitate \& excellentia mirando. ${ }^{123}(H P, 358)$

Die Szene endet mit einer humoristischen Note, die einer allegorischen Deutung zuvorkommt. Die Quelle ist von einem «Vorhängchen » $(H P, 361)$ bedeckt, das mit griechischen Buchstaben als «YMHN ${ }^{124}$ beschriftet ist.

\footnotetext{
$120[\ldots]$ ob all der erörterten Wunder, verharrte ich bestürzt und ganz und gar von Sinnen.

121 Fogliati, L'isola di Citèra, S. 22.

$122[\ldots]$ sah ich auf den ersten Blick ein sehr und in hohem Maße verblüffendes Wunder; [...]

$123[\ldots]$ da wir an diesem Orte zudem diese Neuartigkeit und Unübertrefflichkeit der staunlichen Quellen erschauten.

124 «HYMEN - die Vermählung, deren Gott und Ritus»; sicher auch mit einer sexuellen Komponente. Siehe dazu ReISER, Kommentar, S. $5^{20}$.
} 
Als Poliphilo das Vorhängchen mit dem Pfeil Amors durchschiesst und Venus zum Vorschein kommt, ist er quasi betäubt von ihrem Anblick. Es handelt sich nicht, wie man im ersten Moment erwarten könnte, um eine Statue der Venus, sondern um die lebendige Gottheit - deren Beschreibung als «staunliche Wesenheit » («mirando composito », HP, 362) (25 $^{125}$ allerdings der Beschreibung einer Statue gleicht. Die Darstellung der Göttin setzt mit « Verzückung» und «Bange[n] ( («marauiglia \& terriculo », $H P, 362$ ) ein und endet wiederum mit Staunen und Lob.

Imperoche di incredibile miraueglia stupefacto nellanimo mio summamente la benignitate diuina laudaua. ${ }^{126}(H P, 364)$

Bei Ankunft des Mars verlassen Polia und Poliphilo die Quelle und werden zum Grabmahl des Adonis geleitet. Neben der Gestaltung des Grabes erregt die Statue der Venus am meisten Aufsehen:

Sopra la plana del præfato sepulchro la Diuina Genitrice sedeua puerpera exscalpta, non sencia summo stupore di pretiosa petra Sardonyce tricolore $[\ldots]^{127}(H P, 373)$

Wurde der « Cythereische[] Körper» zuvor in seiner Natürlichkeit gepriesen, wird er jetzt als Werk von «unglaublicher Erfindung und Kunstfertigkeit » gezeichnet $(H P, 362 ; 373)$.

O bellissima operatura da contemplare miraculosa. Solamente del spirito uitale diminuta. ${ }^{128}(H P, 374)$

Die lebendige Venus und die Venusstatue unterscheiden sich kaum im Aussehen und sind sich in der Beschreibung fast gleich, einzig die Schöpfungsund Fertigungsarbeit wird in Bezug auf die Statue betont. Die lebendige Figur geht in die künstliche über - zuerst nur in der Art und Weise der Beschreibung, dann effektiv in der materiellen Form. Das Ende des ersten Buches wird im

125 Reiser übersetzt «composito » mit « Wesenheit», wobei die schöpferische Bedeutung von "comporre“ (zusammenstellen, komponieren, verfassen) verlorengeht. Gerade an dieser Stelle (und im ganzen Werk) ist diese aber zentral.

126 Dennoch pries ich von unglaublicher Verwunderung verblüfft in meinem Sinn in höchstem Maße die göttliche Güte, [...]

127 Auf der Fläche des vorerwähnten Grabmahls saß - nicht ohne mein stärkstes Erstaunen die Göttliche Erzeugerin als Entbindende in kostbarem, dreifarbigem Sardonyxstein ausgehauen, $[\ldots]$

128 Oh wunderschöne Figurengruppe, zu betrachten wunderbarlich! Alleiniglich um den Lebenshauch vermindert! 
Ende des zweiten Buches gespiegelt, wenn Polia als «himmliches Götterbild » $(H P, 377)$ gezeichnet wird.

Der erste und der zweite Teil bestätigen die in der Einleitung angekündigte Erzählstruktur, die vom Staunen bestimmt wird. Die langen Ekphrasen bilden den Kern der beiden ersten Teile, unterbrochen von einzelnen szenischen Episoden wie dem Bad mit den Nymphen, dem festlichen Empfang im Palast der Eleuterilyda oder dem Liebesritus.

\subsubsection{Teil}

Das zweite Buch der $H P$ erzählt in wechselnder Perspektive zum einen von den ersten Begegnungen zwischen Polia und Poliphilo, zum anderen bezeugt es die im Traum entstandene gegenseitige Liebe. Die beiden Liebenden betrachten einander, wobei es nicht mehr Poliphilo ist, der vor Verlangen beinahe zugrunde geht, sondern Polia, die von « ungemäßigter Begierde angetrieben » ist und den Geliebten bestaunt («miraua », $H P, 428$ ).

Et ad gli mei insaciabili \& desiderosi risguardi obiecto delectabile, Dalla uacatione del quale impatiente, \& di auiditate stimulata, \& da immodesto appetito impulsa, \& dassi amorosi oblectamenti capta \& possessa, che quasi externata \& in extasi immobile il miraua $[\ldots]^{129}(H P, 428)$

Poliphilo hingegen ist frei von quälenden Begierden und bezeichnet den Anblick Polias als «Schauspiel der erhabenen und verehrenswerten Zierde Deines wunderschönen Haupts», als «ausgezeichnete[s] und staunliche[s] Bildnis » $(H P, 391)$.

Das zweite Buch handelt von Begegnungen und Ereignissen, es werden keine Kunstwerke mehr beschrieben. Entsprechend finden sich nur noch wenige Staunensäusserungen, das Staunen hat ausserhalb der fantasievollen Sprachbilder und -werke keinen Platz. In gewisser Weise bedeutet es eine einschneidende Abwertung der Liebesgeschichte, wenn die Erzählung im ersten Buch vor lauter Staunen nur so strotzt, die Liebesgeschichte im zweiten Buch aber aus dem Bereich des Staunens ausgeschlossen wird.

Der Roman endet, als Polia verschwindet und Poliphilo erwacht. Was bleibt, sind die schmerzliche Erinnerung an die Umarmung des « seligen Abbildes »

$129[\ldots]$ und meinem unersättlichen und verlangentlichen Schauen als entzückender Gegenstand. Von dessen Entbehren war ich ungeduldig und von Sehnsucht angestachelt, von ungemäßigter Begierde angetrieben, von solch verliebten Ergötzlichkeiten gefangen und besessen, daß ich ihn, gleichsam aus mir getreten in Entrückung unbeweglich anschaute $[\ldots]$ 
sowie ein gemischtes Gefühl aus «staunlicher Süße und eindringlicher Bitterkeit » $(H P, 465)$.

\subsection{Literatur als arché techné}

Kunst, Bildhauerei und Architektur, wie sie im Roman imaginiert und beschrieben werden, sind das primäre Objekt des Staunens und Begehrens. Insbesondere der Architekt - sein Ingenium, sein Schaffen und seine Werke wird wiederholt gepriesen:

Teniua in se tanta cumulatione di miraueglia, che io di stupore insensato staua alla sua consideratione. Et ultra molto piu la immensitate dillopera, \& lo excesso dilla subtigliecia dil opulente \& acutissimo ingiegno, \& dilla magna cura, \& exquisita diligencia dil Architecto. ${ }^{130}(H P, 25)$

Die Pyramide und der Obelisk - von denen an dieser Stelle die Rede ist bergen eine derartige Akkumulation des Wunderbaren, dass Poliphilo «vor Verwunderung von Sinnen dastand » $(H P, 25)$. Über die Immensität des Werkes werden Poliphilo die herausragenden Qualitäten des Architekten bewusst: sein scharfer Geist und die höchstmögliche Sorgfalt, die als übernatürliche, ja göttliche Fähigkeiten erscheinen.

perche questa oltra lo ingegnio humano \& qualunque anaglyptice era faberrimamente facta. ${ }^{131}(H P, 54)$

La ingente cupula monstraua maximo inditio, piu presto de diuina operatione che de humana obstentatione. Ma se humana, Non senza stupore \& accusatione dellambitione de tanto tentato di arte fusoria ad allo ingegno humano, Perche considerando tanta uastitate, in una sola \& solida conflatura, \& iacto de metallo, como io arbitraua, Io rimansi summopere mirabondo \& allucinato, damnandola quasi alla impossibilitate. ${ }^{132}(H P, 201)$

$130[\ldots]$ barg in sich eine solche Zusammenhäufung von Wunderbarem, daß ich vor Verwunderung von Sinnen dastand, da ich sie mir vor Augen führte; und überdies viel mehr die Unermeßlichkeit des Kunstwerkes und der Überschwang der Feinheit des mächtigen und äußerst scharfen Verstandes und der großen Umsicht und ausgesuchten Sorgfalt des Architekten.

131 Denn diese war jenseits der menschlichen Erfindungsgabe und jedweder erhabenen Bildnerei allerkunstreichst ausgeführt.

132 Die gewaltige Kuppel legte gewaltig die Annahme nahe, eher von göttlichem Zustandebringen zu sein denn menschlicher Zurschaustellung; wenn aber menschlich, nicht ohne Verblüffung; und dem menschlichen Geiste ein Merkzeichen des Ehreifers eines großen Wagens in der Schmelzkunst. Denn, wenn man in Betracht zieht, daß solche Weite in 
de diuo excogitato, \& de superba operatura, \& mirandi liniamenti, de stupenda ostentatione, condito mirabilissimo. ${ }^{133}(H P, 214)$

Die Passagen über den Architekten, seine Planungs- und Kompositionsarbeiten, seine geometrische Gestaltung und materielle Fertigung gründen auf der Lektüre von Klassikern der Architekturtheorie, insbesondere der Werke Vitruvs und Albertis. ${ }^{134}$ So schreibt Alberti beispielsweise im Vorwort seiner Zehn Bücher über die Baukunst:

Architectum ego hunc fore constituam / qui certa amirabilique ratione et uia tum mente animoque diffinire: tum et opere absoluere didicerit quecunque ex ponderum motu corporumque compactione et coagmentatione dignissimis hominum usibus bellissime commodentur: Quæ ut possit comprehensione et cognitione opus est rerum optimarum et dignissimarum. ${ }^{135}$

Der Architekt nach Albertis Definition hat gelernt, ein Gebäude zu planen und umzusetzen, das statisch solide ist und eine bestimmte Funktion für Mensch und Gesellschaft erfüllt. Das ästhetische Grundprinzip von Albertis Architekturtheorie ist dasjenige der Schönheit, die insbesondere auf den Prinzipien des consensus (Übereinstimmung), der conspiratio (Einklang), der collocatio

einem einzigen und massiven Ganz- und Metallguss, wie ich vermutete, bewältigt war, blieb ich im höchsten Grade erstaunt und geblendet, da ich sie gleichsam als Unmöglichkeit ausschloß.

$133[\ldots]$ von göttlicher Ersinnung und von stolzer Ausführung und staunlichen Profilierungen, von verblüffender Stattlichkeit, allerwunderbarster Anordnung, [...]

134 Fogliati, Hypnerotomachia Poliphili, S. 17. Liane Lefaivre hat zeigen können, dass in der $H P$ sowohl die Termini Vitruvs als auch die „neueren“ Termini Albertis vorkommen und miteinander vermengt werden. Insbesondere sind einige der Latinismen dem Wortschatz Albertis entlehnt. Lefaivre interpretiert deshalb die $H P$ als Werk Albertis. Ihre These fusst sehr stark auf dem Vokabular, während die gravierenden konzeptionellen Unterschiede aus dem Blickfeld geraten. Siehe LefaIVRe, Leon Battista Alberti, S. 37-38, insbesondere Fn. 385 . Siehe dazu ebenfalls Stefano Borsi, Polifilo architetto. Cultura architettonica e teoria artistica nell'Hypnerotomachia Poliphili di Francesco Colonna (1499), Roma 1995.

135 Leonis Baptiste Alberti de re aedificatoria incipit lege feliciter, Florenz 1485, S. ai: «Ein Architekt wird der sein, behaupte ich, der gelernt hat, mittels eines bestimmten und bewundernswerten Planes und Weges sowohl in Gedanken und Gefühl zu bestimmen, als auch in der Tat auszuführen, was unter Bewegung von Lasten und der Vereinigung und Zusammenfügung von Körpern den hervorragenden menschlichen Bedürfnissen am ehesten entspricht und dessen (möglichste) Erwerbung und Kenntnis unter allen wertvollen und besten Sachen nötig ist. » Die Übersetzung stammt aus LEON BATTISTA Alberti, Zehn Bücher über die Baukunst, ins Deutsche übertragen, eingeleitet und mit Anmerkungen und Zeichnungen versehen durch Max Theuer, Darmstadt 1975, S. 9-10. Die lateinische Version von 1485 ist auf e-rara online verfügbar: https://www.e-rara.ch/ zut/wihibe/content/titleinfo/2825833 (Stand April 2021). 
(Anordnung) und der concinnitas (Harmonie) beruht.136 Das perfekte Gebäude unterstützt den Menschen in seiner moralisch-ethischen Entfaltung, es entspricht seinen Bedürfnissen.

Die architektonischen Werke der $H P$ aber, die ebenfalls dem Prinzip der concinnitas unterliegen, gehen nicht aus der ratio, sondern aus der imaginatio hervor. Sie erfüllen keine funktionalen oder gesellschaftlichen Bedürfnisse, sondern sind dazu da, betrachtet und bestaunt zu werden.

Diese unterschiedliche Ausrichtung auf ratio und imaginatio zeigt sich auch im Umgang mit den antiken Quellen. Alberti beklagt den Mangel an antiken Quellen, die schlechte Überlieferung der Texte Vitruvs sowie den Zerfall der antiken Baustätten - eine Haltung, die sich auch in der HP findet. ${ }^{137}$ Als Mittel zur Überwindung dieser Mängel schlägt Alberti Quellensuche und profundes Studium vor. Colonna hingegen wählt die Imagination, und zwar nicht um die Lücken zu füllen, sondern um den überlieferten literarischen Fundus fantasiereich weiterzuspinnen. Albertis Blick gilt realen Werken, während die $H P$ sich auf den architektonischen Reichtum möglicher fiktiver Werke fokussiert. Und natürlich darf nicht vergessen werden, dass das Staunen bei Alberti zwar gelegentlich auftritt, nie aber eine derart zentrale Rolle spielt wie bei Colonna.

Die Konzeption der Architektur in der HP beinhaltet den poetologischen Kern des Romans. Der Begriff „Architektur“ setzt sich zusammen aus den griechischen Begriffen arché (Anfang, Ursprung) und techné (Kunstfertigkeit); insofern ist die Architektur als „Handwerk des Ursprungs“, als „erste Kunst“ zu verstehen. Der Architekt und der Schriftsteller sind Schöpfer von Neuem, noch nie Dagewesenem, mittels erstaunlicher Imagination und Schaffenskraft.

\subsection{Stupore und admiratione}

In der $H P$ werden die unterschiedlichen Staunensbegriffe sehr prominent und nicht selten gemeinsam verwendet.

Et in loco dele trece capreolate cum uiuace \& ingente spire miraua stupente gli uiperi \& intortigliati serpi. ${ }^{138}(H P, 27)$

136 Günther Fischer, Leon Battista Alberti. Sein Leben und seine Architekturtheorie, Darmstadt 2012, S. 180-181.

137 Alberti, Zehn Bücher über die Baukunst, S. 291.

138 Und am Ort der wildverrankten Flechten schaute ich mit lebensechten und gewaltigen Spiralen die erstaunlichen Vipern und eingedrehten Schlangen, [...] 
Si gli fragmenti dilla sancta antiquitate \& rupture \& ruinamento \& quodammodo le Scobe ne ducono in stupenda admiratione, \& ad tanto oblectamento di mirarle, quanto farebbe la sua integritate? ${ }^{139}(H P, 59)$

Et in momento dal profundo dil tristo core trahendo gli gemitosi sospiri, nella tenace reminiscentia replicaua quanto piacere \& dilecto in puncto haueano gli sensi mei perdito, Imperoche quella operatura era piena di meraueglia, \& di stupore. $^{140}(H P, 67)$

Io alhora sentiui gli spiriti mei stupefacti, mirabondo $[\ldots]^{141}(H P, 148)$

Die gemeinsame Verwendung verschiedener Staunensbegriffe kann als Klimax gelesen werden. Sie unterstreicht die ausserordentliche, kaum beschreibbare Qualität des Betrachteten und bringt alle Dimensionen des Staunens ein: das verblüffte Staunen, das bewundernde Staunen, die visuelle Komponente des Staunens und die Verwunderung.

Stupor und ( $a d$-)miratio sowie die entsprechenden verbalen und adjektivischen Formen können zur Charakterisierung sowohl des Betrachteten als auch des Betrachtenden verwendet werden und bezeichnen immer eine Interaktion des Sehenden mit dem Gesehenen. Stupor bringt die Konnotation der Verblüffung mit ein, sowohl der Sinne wie auch des Intellekts, ${ }^{142}$ zudem ist er teilweise gepaart mit Furcht und Schrecken. Demgegenüber betont ( $a d$-)miratio die Qualität und die Wirkung des Sehens und Betrachtens.

Neben stupor sind es vor allem die adjektivischen Formen (ob-)stupefacto, stupido, stupefacente, stupendo und stupente, die zur Anwendung kommen. Das verblüffte Staunen wird nie als Verb, als Tätigkeit, sondern immer nur als Zustand bzw. Eigenschaft eines Zustands bezeichnet. Während die ersten beiden adjektivischen Formen den Betrachter charakterisieren, können die letzten drei Varianten sowohl für die Charakterisierung des Betrachters («miraua stupente», «schaute ich [verdutzt]», HP, 27) als auch für die

139 Wenn die Trümmer des verehrungswürdigen Altertums und Bruchstücke und Einstürze, gewissermaßen die Sägespäne davon, in uns zu solch erstaunlicher Bewunderung und so Ergötzen, sie zu schauen, verleiten, wie viel bewirkte ihre Unversehrtheit?

140 Und da ich augenblicklich die stöhnenden Seufzer aus der Tiefe des traurigen Herzens zog, wiederholte sich in der hartnäckigen Wiedererinnerung genau, wie viel an Gefallen und Entzücken meine Sinne verloren hatten, aus dem Grunde, daß jenes Bauwerk voll von Verwunderung war und von Staunen.

141 Danun gewahrte ich, wie meine Geister wie gebannt waren, da ich mich wunderte, [...]

142 Vgl. dazu $H P, 17$ («gia obstupefacto lo intellecto, \& sopito lappetito », « indes der Verstand gleichsam sinnberaubt und bereits erstarrt war, und eingeschläfert die Begier»), ebenso $H P, 92$ («quasi io rimansi degli sensi [...] stupido », « verblieb ich gleichsam von Sinnen [...] erstaunt ») oder $H P, 355$ (« allo intellecto ostupire», « verblüffte den Verstand »). 
Charakterisierung des Betrachteten («uno stupendo Cubo », « ein erstaunlicher Kubus », $H P$, 24) eingesetzt werden.

Im Wortfeld $\operatorname{der}(a d$-)miratio wird admiratio als Substantiv verwendet, wobei auch hier die adjektivischen und verbalen Formen deutlich häufiger auftreten als das Substantiv. Admiratio finden wir in Verbindungen wie «WÜRDIG DER BEWUNDERUNG » («digne di admiratione », HP, 20, 50, 174) oder « voller Verwunderung » («cum admiratione», $H P, 18$ ) in adverbialer oder adjektivischer Bedeutung, wenn beispielsweise das Sehen begleitet und charakterisiert wird $(H P, 228)$. Admiratio wird sowohl im Sinne von Bewunderung - insbesondere der Bewunderung des Künstleringeniums oder eines kunstreich gefertigten Werkes - als auch in der Bedeutung von Staunen verwendet. Folgende Zitate veranschaulichen im ersten Beispiel die Bewunderung, im zweiten die synonymische Verwendung von stupor und admiratione:

Vorei pero che tu sapesti, che non senza grande admiratione di humano ingegno \& cum ardente studio \& incredibile diligentia fue fabricata quella ingente machina, Cum perplexibilitate dello intellecto ad intendere il suo diuino concepto. ${ }^{143}(H P, 132)$

Per laquale cosa non manco piacere \& dilecto cum stupore quiui tali solenni riti \& celebre feste me inuase, che la admiratione degli præcedenti triumphi. ${ }^{144}$ $(H P, 195)$

Bisweilen wird admiratio auch als Eigenschaft eines Kunstwerkes bezeichnet:

[...] gli gradi di mirabile tessellatura offeriuase di grande admiratione, \& ecessiuamente delectabile artificio, sencia dubio di fatichare omni humana inuitione \& senso. ${ }^{145}(H P, 316)$

Die «tessellatura » ist von «großer Bewunderungswürdigkeit» $(H P, 316)$, das Bewunderungswürdige ist der «Einlegearbeit» $(H P, 316)$ als Eigenschaft eingeschrieben. « $[\mathrm{A}]$ dmiratione» wird in diesem Zitat als sinnesbetäubende

143 Ich möchte freilich, daß Du wissest, daß jene gewaltige Konstruktion nicht ohne großes Augenmerk des menschlichen Geistes und mit loderndem Eifer und unglaublicher Umsicht gefertigt ward, um beim Verstehen ihres göttlichen Entwurfes für die Verwirrung des Verstandes zu sorgen.

144 Aus jenem Grunde überkam mich allhier nicht geringes Vergnügen und Ergötzen, mit Verblüffung über solch festlicher Gebräuche und solch preiswürdiger Feste, als es bei den vorausgegangenen Triumphen Bewunderung gewesen war.

$145[\ldots]$ von staunlicher Einlegearbeit, von großer Bewunderungswürdigkeit und überschwenglich entzückender Kunstfertigkeit [...], welche zweifelsohne jede menschliche Betrachtung und Sinneswahrnehmung überforderten. 
Eigenschaft genannt und kann so wiederum als Synonym zu stupor gelesen werden. Auch das Adjektiv admirando taucht gemeinsam mit adjektivischen Formen von stupor auf:

Il quale [Amor] conobi per coniectura mirabonda, \& esso di tanto opificio di Polia la raritate admiranda, \& la magnitudine di tante uirtute \& bellece stupenda, pensando io uerisimelmente, che alla sua bellissima psyche nel animo non sencia concupiscentia piu uenusta, piu prestante, $\&$ di piu eminente excellentia lantiponeua. ${ }^{146}(H P, 275)$

Interessant an diesem Zitat ist insbesondere, dass alle Staunensadjektive («mirabonda», «admiranda», «stupenda») auf Polia bezogen sind und nicht auf Amor, den Betrachter. Die erstaunliche Schönheit Polias wird so absolut gesetzt - mit anderen Worten: Dass Polia erstaunlich schön ist, hängt nicht vom Urteil des Betrachters ab, sondern ist fest gegeben. Alle drei Adjektive werden synonymisch verwendet und erscheinen als variantenreiche Beschreibung ein und derselben Qualität. Als Qualitätsmerkmal finden wir das Erstaunliche auch in Bezug auf (Bau-)Werke («admiranda piramide», « phantastische Pyramide», HP, 23) oder aber als Konnotation von Poliphilos Denken («admiranda contemplatione», « erstaunliche Betrachtungen», $H P, 53)$.

Die am meisten verwendete Form aus dem Begriffsfeld der admiratio ist das Verb mirare. « Mirai » - seltener auch « ammirai » oder « rimirai » - taucht unzählige Male auf und kennzeichnet die Grundhaltung Poliphilos: Staunend betrachtet er, was ihm in der Traumwelt begegnet. An einigen Stellen ist mirare zusätzlich qualifiziert:

cum grande piacere mirai ${ }^{147}(H P, 95)$

mirai attonito ${ }^{148}\left(H P, 15^{8}\right)$

cupidamente mirai ${ }^{149}(H P, 433)$

146 Von jenem, so glaubte ich es zu verstehen, da auch er Polia von solcher Kunstarbeit schaute, wobei er die Feingliedrigkeit bewunderte und das Ausmaß solcher Tugenden und verblüffenden Schönheiten, dachte ich mit gewisser Wahrscheinlichkeit, daß er sie in Gedanken nicht ohne Verlangen seiner wunderschönen Psyche als reizendschönere, vortrefflicher und, als von vornehmlicher Vortrefflichkeit, voranstellte.

$147[\ldots]$ schaute ich dann mit grossem Vergüngen, $[\ldots]$

$148[\ldots]$ alsdann schaute ich, erschüttert, [...]

$149[\ldots]$ als ich Dich begehrlich schaute $[\ldots]$ 
«Mirai» kennzeichnet nicht nur die Tätigkeit Poliphilos, sondern nimmt zudem eine narrative Funktion ein. Der Text erzählt das, worauf Poliphilos Augen sich richten. Gleichzeitig handelt es scih um eine Markierung für den Leser: „Achtung, da folgt etwas Neues, Ausserordentliches, Staunensund Nennenswertes!" Das innere Auge des Lesers identifiziert sich mit dem «mirai » Poliphilos und nimmt diesen Blick aus Leserperspektive ein.

Das Adjektiv mirabile wird attributiv (« tale mirabile composito », « folgende staunliche Zusammenfügung », $H P$, 55; « una mirabile \& egregia fontana», « einen ausnehmlichen Brunnen», $H P, 88)$ oder als Adverb der kunstfertigen Herstellung («mirabilmente exscalpte», «auf staunenswerte Weise ausgehauen», $H P, 93)$ genannt. Als Synonyme werden zudem miro, mirando und mirifice verwendet. Wie schon bei Giovanni Pontano ist die Qualität des Wunderbaren ein Pendant zur staunenden Betrachtung:

Nella tabella dextra mirai $[\ldots]$ uno mirabile pallacio. ${ }^{150}(H P, 163)$

Mirare und mirabile entstammen derselben lateinischen Wurzel - mirus -, sie bilden zwei Perspektiven (Betrachter und Betrachtetes) auf ein und dasselbe Phänomen. Aus diesem Wortstamm ist auch meraviglia hervorgegangen, das Wort geht als Substantiv, Adjektiv und Verb auf mirabilia und mirabilis zurück. Die Begriffe rund um meraviglia erscheinen in der HP in unterschiedlicher Schreibweise - miraveglia, maraveglia, meraviglia -, wobei die heutige Schreibweise die seltenste ist. Das Substantiv tritt nur wenige Male und gemeinsam mit einem weiteren Staunensbegriff auf:

Teniua in se tanta cumulatione di miraueglia, che io di stupore insensato staua alla sua consideratione. ${ }^{151}(H P, 25)$

Impero di incredibile miraueglia stupefacto nellanimo mio summamente la benignitate diuina laudaua. ${ }^{152}(H P, 364)$

Im ersten Fall ist es eine Anhäufung, im zweiten Fall eine unglaubliche miraveglia, die als Auslöser des Staunens genannt wird. Einmal sind es die Sinne, die ausser Kraft gesetzt werden, einmal ist es der Geist, der von ihr übermannt wird. Auch das Adjektiv taucht in Kombination mit stupore auf, in diesem Fall mit einer differenzierenden Wirkung:

150 Auf der rechten Tafel schaute ich [...] eine[n] staunlichen Palast[] [...]

$15^{1}[\ldots]$ barg in sich eine solche Zusammenhäufung von Wunderbarem, daß ich vor Verwunderung von Sinnen dastand, da ich sie mir vor Augen führte; [...]

$15^{2}$ Dennoch pries ich von unglaublicher Verwunderung verblüfft in meinem Sinn in höchstem Maße die göttliche Güte [...] 
[...] da miraueglioso stupore circumuenuto [...] grandemente sospeso me ritrouai, $[\ldots]^{153}(H P, 185)$

Miraveglia, miraveglioso und miravegliosamente werden als Synonyme zu miracolo, miraculoso und miraculosamente verwendet. Ein Wunder genauso wie ein Kunstobjekt können als miraveglia oder miraculo bezeichnet oder mit den Adjektiven entsprechend charakterisiert werden. Das Verb miravegliare (ebenso maravegliare) wird nur vereinzelt verwendet, vorwiegend um Verwunderung auszudrücken. So wundern sich beispielsweise die Sinnesnymphen darüber («MiRAVEgLiAntise», HP, 94), weshalb und wie Poliphilo in ihr Reich gelangen konnte. Ebenfalls wundert sich Eleuterilyda über die Geschichte Poliphilos, als er erzählt, was er auf dem Weg zu ihrem Palast alles gesehen und überstanden habe $(H P, 101)$. In beiden Beispielen hat miravegliare keine ästhetische Konnotation und unterscheidet sich dadurch klar von mirare. Im Vergleich zu stupor und admiratio werden die Begriffe rund um miraveglia viel weniger häufig verwendet - ich würde begrifflich deshalb nicht von einer Poetik der meraviglia, sondern viel eher von einer Poetik der Verblüffung (stupor) und des staunenden Betrachtens (admiratio) reden.

Das Begriffsfeld um stupor und admiratio wird unterstützt durch folgende Adjektive, die meist in superlativer Form zum Ausdruck kommen: eximio (esimio), egregio, divino, ingegnoso, sublime, eccellente und portentoso. ${ }^{154}$ Weiter finden wir ergänzende Begriffe des Vergnügens wie lepido und commendato ${ }^{155}$ beide ebenfalls meist in der gesteigerten Form. Auch das Schöne erhält neben dem Attribut des Staunenswerten und Wunderbaren weitere Qualitäten: Prcestante (prestante), venusto und formoso ${ }^{156}$ werden häufig benutzt und zeugen einerseits von der herausragenden Qualität, zum anderen von der harmonischen und grazilen Form. Die Kunstfertigkeit des Architekten wird mit Begriffen wie fabberimo ${ }^{157}$ bekräftigt. Nicht ohne Bedeutung ist es, dass ein Grossteil dieser Adjektive lateinische Begriffe sind, die der Autor für seinen Roman italianisiert hat. Bewundert und bestaunt werden nicht die zeitgenössischen Werke und Künstler - im Gegenteil, deren Stil- und Kunstlosigkeit wird bedauert -, sondern ausschliesslich diejenigen der Antike. Der antiken Kunst und Architektur in ihrer vom Autor imaginierten Form wird mit den italianisierten Staunensbegriffen eine eigene Sphäre geschaffen.

\footnotetext{
$153[\ldots]$ von wunderbarlicher Verblüffung überkommen [...] war mir gewaltiglich bange, [...]

154 Hervorragend, aussergewöhnlich, göttlich, einfallsreich, erhaben, herausragend, wunderbar.

155 Geistreich, lobenswert.

156 Rüstig, lebendig, anmutig, üppig.

157 Geschickt, kunstfertig.
} 


\subsection{Konklusion}

Die $H P$ konfrontiert uns mit verschiedenen Rollen und Funktionen, die das Staunen in Literatur und Kunst übernehmen kann. Zuallererst ist es die narrative Funktion des Staunens, die als Basis des Romans betrachtet werden kann: Erzählt wird, worüber gestaunt wird. Das Staunen bestimmt, zumindest im ersten Buch, den narrativen Fortgang. Zudem hebt es die Übergänge zwischen verschiedenen Bereichen und Passagen hervor, indem es einen Moment des Innehaltens, Erinnerns und Beschreibens konstituiert.

Der Blick Poliphilos richtet sich ausschliesslich auf fingierte Artefakte seien dies Gebäude, Skulpturen, Kleider, Bilder, Gärten, Riten oder sogar die Figuren. Die Fiktion scheint, wie schon bei Pontano, den erstaunlichen Charakter des Erzählten zu befördern. Sie geht, wie in der Einleitung der $H P$ beschrieben, aus dem Staunen hervor - dem Staunen kommt damit eine imaginativ-poietische Rolle zu.

Die betrachteten Werke folgen entweder klassischen Kunstnormen wie Harmonie und Proportionalität oder aber orientieren sich an lebendigorganischen Formen, die der Natur zwar ähnlich sind, deren Ordnung aber zugleich sprengen. Der staunende Blick animiert dazu, die Imaginationskraft sowie die Kunstfertigkeit des Künstlers - sei dies ein Bildhauer, Maler, Architekt oder, metaliterarisch betrachtet, der Schriftsteller - zu ergründen. Dazu werden die Kunstwerke ausführlich beschrieben, wobei die Beschreibungen mit einem staunenden Gestus eingeleitet und nach sachlich-technischen Erläuterungen von einem erneuten Ausdruck des Staunens bekräftigt werden. Das Staunen bildet gewissermassen die Klammer der Ekphrasen. Gleichzeitig wird das Erstaunliche als zentrale Eigenschaft der Kunstwerke erkannt; es ist den Werken eingeschrieben, verankert im Ausdruck der künstlerischen Idee und der Art und Weise der Fertigung.

Mit der Ausrichtung des Staunens auf die Kunst und den Künstler hängt zusammen, dass das Objekt der Liebe nicht als Mensch, sondern als Kunstwerk im Blickpunkt steht. Mehr und mehr verwandeln sich Polia und Venus als Urform der Liebe und des Schönen - von einem lebendigen Wesen in ein Abbild bzw. eine Statue. Das Staunen besitzt eine transformative Kraft hin zum Ästhetischen.

Im Gegensatz zu Poliziano und Pontano, die mit ihren Texten eine Poetik des Staunens formuliert, das heisst das Staunen als übergeordnetes Ziel der Dichtung definiert, es als (mythologischen) Ursprung der Dichtung konstituiert und die poetischen Mittel in seinen Dienst gestellt haben, konstituiert sich die $H P$ in erster Linie als Ästhetik des Staunens. Diese steht für die Interdependenz zwischen dem Künstler, dem Kunstobjekt und dem Betrachter, die über das 
Staunen hergestellt wird. Wie bereits von Pontano und Poliziano beschrieben, ist das Staunen eine Bewegung, die sich als Übertragung vom Dichter und vom Erzähler - die selbst aus dem Staunen kommen - auf den Text und von diesem auf den Leser vollzieht. Diese Bewegung scheint mir für eine Poetik wie für eine Ästhetik des Staunens zentral zu sein, denn sie offenbart in ihrem Kern die Funktionsweise des Staunens.

Während der enkomiastische Kontext, die von Poliziano und Pontano bevorzugten Mittel der variatio und der kreativen imitatio wie auch die ethische und zivilisatorische Funktion der Dichtung für Colonna keine Rolle spielen, zeigen sich doch Überschneidungen in den Kernpunkten der drei Poetiken bzw. Ästhetiken. Da ist zuallererst die Betonung der Fiktion, die für alle drei Autoren von besonderer Bedeutung ist, da sie zum einen die Imaginationskraft des Künstlers und des Poeten ausstellt, zum anderen die Inhalte mit staunenswerten Elementen anreichert. Letzteres ist zudem gekoppelt an den Charakter des Neuen und Überraschenden, der mit den fingierten Inhalten einhergeht. Als Beispiele für das ausserordentliche Mass an Fiktion bei allen drei Autoren seien die Mythologisierung Giulio de' Medicis in den Stanze per la giostra, die Personifizierung der neapolitanischen Region in Lepidina oder aber die Traumwelt in Colonnas $H P$ genannt. Allerdings bezieht sich die fictio nicht nur auf den Inhalt, sondern auch auf die literarische Ausdrucksform - so sind auch die Genreinnovationen, die von Poliziano, Pontano und Colonna gleichermassen getätigt werden, im Rahmen einer Betonung der Fiktion zu verstehen.

Eine weitere Analogie zeigt sich in der strukturgebenden Funktion des Staunens innerhalb der Erzählung. Sowohl die Stanze, Lepidina wie auch die $H P$ lassen ihre Erzählungen den staunenden Blicken ihrer Protagonisten folgen. Mit diesem Punkt sind zugleich zwei weitere Gemeinsamkeiten angesprochen: die Betonung des bildschaffenden Charakters der Sprache und die Zusammengehörigkeit von Sehen und Staunen. Letzteres wird durch die bevorzugte und häufige Verwendung von mirare und admiratio bewerkstelligt, die bildgebende Kraft dichterischer Sprache zeigt sich wiederum in den Ekphrasen der Stanze, den lebendigen Nacherzählungen der Silvae, der visuell (und olfaktorisch) übersprühenden Lebenswelt in Lepidina und De hortis Hesperidum. In ihrer Häufigkeit sowie ihrer Konzentration bildet die Bildlichkeit der Sprache in der $H P$ sicherlich einen Höhepunkt; zudem verdichtet sie sich in einem ausschliesslich ästhetischen Kontext, während die anderen beiden Autoren ihre staunenswerten Inhalte aus mythologischen oder mythologisch inspirierten Handlungen gewinnen. 